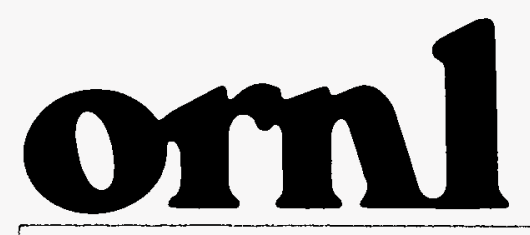

OAK RIDGE NATIONAL LABORATORY

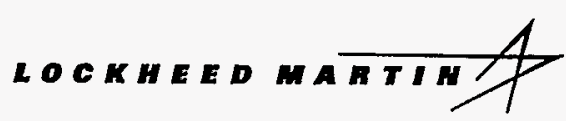

RECEIVED

JAN $O 21997$

ORNLTM-13347

\title{
DCFPAK: Dose Coefficient Data File Package for Sandia National Laboratory
}

K. F. Eckerman

R. W. Leggett

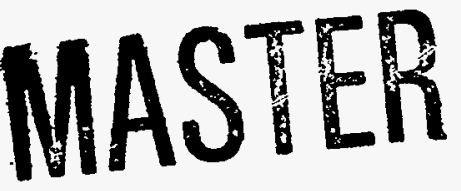


This report has been reproduced directly from the best available copy.

Available to DOE and DOE contractors from the Office of Scientific and Technical Information, P.O.Box 62, Oak Ridge, TN 37831; prices available from (423) 576-8401, FTS 626-8401.

Available to the public from the National Technical Information Service, U.S. Department of Commerce, 5285 Port Royal Rd., Springfield, VA 22161

This report was prepared a an account of work sponsored by an agency of the Uniled States Government. Neither the United States Government nor any agency thereof, nor any of their employees, makes any warranty, express or implied, or assumes any legal liability or responsibility for the accuracy, completeness, or usefulness of any information, apparatus, product, or process disclosed, or represents that its use would not infringe privately owned rights. Reference herein to any specific commercial product, process, or service by trade name, trademark, manufacturer, or otherwise, does not necessarily constitute or imply its endorsement, recommendation, or favoring by the United States Govemment or any agency thereof. The view and opinions of authors expressed herein do not necessarily state or reflect those of the UnitedStatesGovemment or any agency thereof. 


\section{DCFPAK: Dose Coefficient Data File Package for Sandia National Laboratory}

K.F. Eckerman and R.W. Leggett Health Sciences Research Division

Oak Ridge National Laboratory

Manuscript Completed

July 31, 1996

Prepared for Sandia National Laboratory,

Albuquerque, NM 87185 , under contract AS-2472

Prepared by the Oak Ridge National Laboratory Oak Ridge, Tennessee 37831-6285 managed by

LOCKHEED MARTIN ENERGY RESEARCH CORP. for the U.S. DEPARTMENT OF ENERGY under contract DE-AC05-960R22464. 


\section{DISCLAIMER}

Portions of this document may be illegible in electronic image products. Images are produced from the best available original document. 


\section{CONTENTS}

ACKNOWLEDGEMENT $\ldots \ldots \ldots \ldots \ldots \ldots \ldots \ldots \ldots \ldots \ldots \ldots \ldots \ldots \ldots \ldots \ldots$ iii

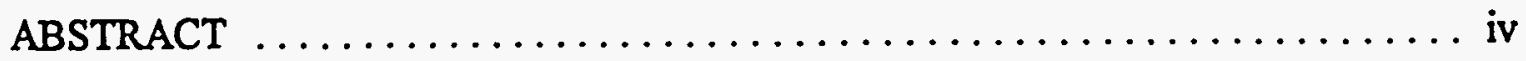

INTRODUCTION $\ldots \ldots \ldots \ldots \ldots \ldots \ldots \ldots \ldots \ldots \ldots \ldots \ldots \ldots \ldots \ldots \ldots$

DATA FILES IN DCFPAK $\ldots \ldots \ldots \ldots \ldots \ldots \ldots \ldots \ldots \ldots \ldots \ldots \ldots \ldots \ldots \ldots \ldots$

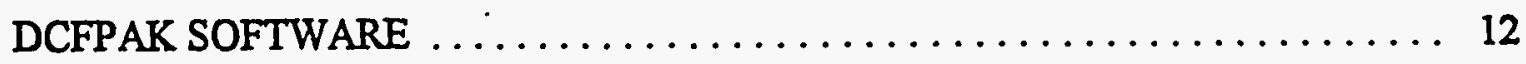

CONNECTING DCFPAK TO THE USER'S DRIVER CODE $\ldots \ldots \ldots \ldots \ldots 16$

INPUT TO THE USER'S DRIVER CODE $\ldots \ldots \ldots \ldots \ldots \ldots \ldots \ldots \ldots \ldots, 17$

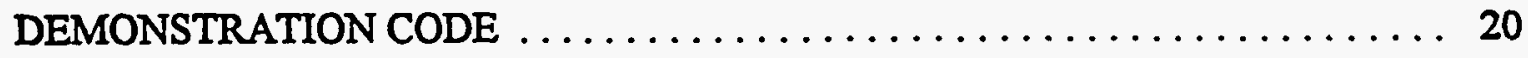

INSTALLATION ON UNIX WORKSTATIONS $\ldots \ldots \ldots \ldots \ldots \ldots \ldots \ldots, 21$

REFERENCES $\ldots \ldots \ldots \ldots \ldots \ldots \ldots \ldots \ldots \ldots \ldots \ldots \ldots \ldots \ldots \ldots \ldots \ldots \ldots \ldots \ldots$

APPENDIX A: Source Code Listings $\ldots \ldots \ldots \ldots \ldots \ldots \ldots \ldots \ldots \ldots$ 


\section{ACKNOWLEDGEMENT}

The work described in this report was performed at Oak Ridge National Laboratory and was supported by Sandia National Laboratory under contract AS-2472. Earlier activities at ORNL in development of the dosimetric data files addressed here were sponsored by the Department of Energy, the Nuclear Regulatory Commission, and the Environmental Protection Agency. The support of these organizations has been critical to the development of our dosimetry research program and is gratefully acknowledged.

The authors are grateful for the contributions of David B. Clauss, Sandia National Laboratory, whose insights into the dosimetric needs in consequence analysis provided the stimulus for this work, and to Barbara Clark, Oak Ridge National Laboratory, for her assistance in the preparation of the final manuscript for publication. 


\begin{abstract}
The FORTRAN-based computer package DCFPAK (Dose Coefficient File Package) has been developed to provide electronic access to the dose coefficient data files summarized in Federal Guidance Reports 11 and 12. DCFPAK also provides access to standard information regarding decay chains and assembles dose coefficients for all dosimetrically significant radioactive progeny of a specified radionuclide. DCFPAK was designed for application on a PC but, with minor modifications, may be implemented on a UNIX workstation.
\end{abstract}




\section{INTRODUCTION}

The U.S. Environmental Protection Agency (EPA) has issued a series of "Federal Guidance" reports that provide the dosimetric information needed by government agencies to implement radiation protection programs in a consistent and adequately protective manner. This information is mainly in the form of "dose coefficients", or estimates of dose per unit exposure to individual radionuclides. These dose coefficients allow the user to relate concentrations of radionuclides in air, water, food, or soil to guidance for radiation dose to workers or members of the public.

Two Federal Guidance reports are currently in use. Federal Guidance Report No. 11 - (Eckerman et al. 1988) provides dose coefficients, in the form of 50-year integrated dose equivalents, for acute ingestion or acute inhalation of radionuclides, based on the biokinetic and dosimetric models of ICRP Publication 30 (1979, 1980, 1981, 1988). Federal Guidance Report No. 12 (Eckerman and Ryman 1993) provides dose coefficients, in the form of dose per unit time-integrated exposure, for external exposure to radionuclides in air, water, or soil, based on state-of-the-art methods in external radiation dosimetry.

The tabulations in these two Federal Guidance reports include dose coefficients for the radionuclides considered in ICRP Publication 30, Limits for Intakes of Radionuclides by Workers (ICRP 1979, 1980, 1981, 1988). For each radionuclide and exposure mode, dose coefficients are provided for the seven organs or tissues assigned risk weighting factors in ICRP Publication 26 (1977): breast; lung; red marrow; bone surface; thyroid; gonads, representing the higher of the coefficients for ovaries and testes; and "remainder", representing the five remaining tissues receiving the highest doses. The tabulations include the effective dose equivalent, $\mathrm{H} \_\mathrm{E}$, derived from the risk weighting factors and equivalent doses for these organs. In Federal Guidance Report No. 12, dose coefficients are also provided for skin because it is frequently the most highly irradiated organ for external exposure.

The software and data package DCFPAK (Dose Coefficient File Package) has been developed to allow electronic access to the full set of dose coefficients summarized in these two Federal Guidance reports and to facilitate consideration of dose coefficients for chains of radionuclides. In addition to the published dose coefficients, the DCFPAK libraries includes dose coefficients for 18 organs not addressed in Federal Guidance Report No. 11, and 17 organs not addressed in Federal Guidance Report No. 12 (Table 1). The additional dose coefficients were generated during the development of these two Federal Guidance reports but were excluded from the published tables to make the tables easier to use and to keep the reports at a reasonable length. For each radionuclide and exposure mode, the DCFPAK libraries include both the effective dose equivalent, H_E, based on the tissue weighting factors given in ICRP Publication 26 (1977), and the effective dose, E, based 
Table 1. Tissues addressed in DCFPAK, Federal Guidance No. 11, and Federal Guidance No. 12.

\begin{tabular}{|c|c|c|c|c|}
\hline \multirow[b]{2}{*}{ Tissue } & \multirow{2}{*}{$\begin{array}{l}\text { Abbreviation in } \\
\text { DCFPAK } \\
\text { libraries }\end{array}$} & \multicolumn{3}{|c|}{ Listed in: } \\
\hline & & DCFPAK & $\begin{array}{l}\text { Federal Guidance } \\
11\end{array}$ & $\begin{array}{l}\text { Federal Guidance } \\
12\end{array}$ \\
\hline Adrenal & Adrenal & Yes & No & No \\
\hline Urinary Bladder Wall & Bld Wall & Yes & No & No \\
\hline Bone Surface & B Surface & Yes & Yes & Yes \\
\hline Brain & Brain & Yes & No & No \\
\hline Breast & Breast & Yes & Yes & Yes \\
\hline Esophagus & Esophagu & Yes & No & No \\
\hline Stomach Wall & St Wall & Yes & No & No \\
\hline Small Intestine Wall & SI Wall & Yes & No & No \\
\hline Upper Large Intestine Wall & ULI Wall & Yes & No & No \\
\hline Lower Large Intestine Wall & LLI Wall & Yes & No & No \\
\hline Kidney & Kidney & Yes & No & No \\
\hline Liver & Liver & Yes & No & No \\
\hline Lung & Lung & Yes & Yes & Yes \\
\hline Muscle & Muscle & Yes & No & No \\
\hline Ovaries & Ovaries & Yes & No & No \\
\hline Testes & Testes & Yes & No & No \\
\hline Gonad" & (Not used) & No & Yes & Yes \\
\hline Pancreas & Pancreas & Yes & No & No \\
\hline Red Marrow & RMarrow & Yes & Yes & Yes \\
\hline Skin & Skin & Yes & No & Yes \\
\hline Spleen & Spleen & Yes & No & No \\
\hline Thymus & Thymus & Yes & No & No \\
\hline Thyroid & Thyroid & Yes & Yes & Yes \\
\hline Uterus & Uterus & Yes & No & No \\
\hline Remainder & (Not used) & No & Yes & Yes \\
\hline
\end{tabular}

Represents multiple tissues considered explicitly in DCFPAK. 
on the newer tissue weighting factors given in ICRP Publication 60 (1991). Tissue weighting factors recommended in ICRP Publication 26 and Publication 60 are compared in Table 2.

The DCFPAK libraries contain dose coefficients for each of nine modes of exposure to a given radionuclide: (1) ingestion; (2) inhalation; (3) submersion, meaning external exposure to (radiations emitted from) the radionuclide in air; (4) immersion, meaning external exposure to the radionuclide in water (from swimming); (5) external exposure to the radionuclide on the ground surface; and (6-9) external exposure to the radionuclide distributed to a depth of $1 \mathrm{~cm}$, a depth of 5 $\mathrm{cm}$, a depth of $15 \mathrm{~cm}$, or an infinite depth in soil. The dose coefficients for internal exposures are 50$\mathrm{y}$ integrated doses to organs following an acute intake and are given in units of $\mathrm{Sv} \mathrm{Bq}^{-1}$. The dose coefficients for external exposures are dose per unit time-integrated exposure. Dose coefficients for external exposures are in terms of Sv per Bq-s per unit volume of environmental medium (liter of air for submersion, liter of water for immersion, $\mathrm{m}^{2}$ of soil for ground surface contamination, and $\mathrm{m}^{3}$ of soil for subsurface contamination of soil).

The dose coefficient for a given radionuclide and exposure scenario does not reflect ingrowth of chain members in the environment, but dose coefficients for ingestion and inhalation do reflect the contribution to dose from ingrowth of chain members in the body after intake of the parent radionuclide. For either internal or external exposure to a radionuclide, DCFPAK determines the radionuclide decay chain (if any) of that radionuclide, truncates the chain after the last potentially significant decay chain member, and assembles the dose coefficients for all potentially significant chain members. For internal exposure, DCFPAK truncates a chain if the cumulative energies for alpha, electron, and photon radiation over a 100-year period are changed less than $1 \%$ by the addition of subsequent chain members. The same procedure is followed for external exposures but with consideration restricted to electrons and photons.

The DCFPAK package, which is written in FORTRAN 77, was developed for use as a module of a radiological assessment package currently used at Sandia National Laboratory. However, DCFPAK is generic in nature and should be compatible with other FORTRAN-based radiological assessment packages. It was designed for use on a personal computer or work station and can be run interactively or in batch mode. 
Table 2. Tissue weighting factors given in ICRP Publication 26 (1977) and ICRP Publication 60 (1991) .

\begin{tabular}{lcc}
\hline & \multicolumn{2}{c}{ Tissue weighting factor $\left(w_{\text {工 }}\right)$} \\
\cline { 2 - 3 } Organ or tissue & ICRP Pub. 26 & ICRP Pub. 60 \\
\hline Gonads & 0.25 & 0.20 \\
Bone marrow (red) & 0.12 & 0.12 \\
Colon & - & 0.12 \\
Lung & 0.12 & 0.12 \\
Stomach & - & 0.12 \\
Bladder & - & 0.05 \\
Breast & 0.15 & 0.05 \\
Liver & - & 0.05 \\
Oesophagus & - & 0.05 \\
Thyroid & 0.03 & 0.05 \\
Skin & - & 0.01 \\
Bone surface & 0.03 & 0.01 \\
Remainder & 0.30 & 0.05 \\
\hline & &
\end{tabular}

2The values were developed for a reference population of equal numbers of both sexes and a wide range of ages. In the definition of effective dose they apply to workers, to the whole population, and to either sex. 
For illustrative purposes DCFPAK was linked to a sample "driver" code called READEM (source code, READEM.FOR and executable file, READEM.EXE). READEM allows an interactive examination of DCFPAK's output for any of the 9 exposure modes and approximately 800 radionuclides addressed in this package.

The DCFPAK package consists of the following data libraries (data files) and software files:

1. nine libraries of dose coefficients, corresponding to the nine exposure modes listed above;

2. a library of information on the half-lives, modes of decay, branching fractions, and decay products of all 838 radionuclides considered in these two Federal Guidance reports;

3. a "data access" module that accesses the 10 data files described above and returns the relevant information;

4. an include file containing information on dimensioning of arrays in DCFPAK;

5. two include files containing common blocks;

6. an include file that provides unit designations to be reserved for IO used by DCFPAK;

7. an "initialization" file that informs DCFPAK of the location of all data files and their record lengths;

8. an illustrative driver code that serves the dual purpose of allowing an examination of available DCFPAK output and illustrating how to establish communication between the user's driver code and DCFPAK's data access module.

To access DCFPAK; the user must insert the following into his driver code: include statements that reference the files described in items 4-6 above, and a call statement, described later, that transfers the input variables describing the radionuclide, particle size, desired output, and other case-specific information to DCFPAK. The user's code and the source code DCFPAK.FOR must then be compiled and the resulting object files must be linked. 


\section{DATA FILES IN DCFPAK}

Ten data files are included in DCFPAK. The data files are all "formatted direct-access files" consisting of ASCI characters, with all records in a given file of the same length and each record ending with an ASCII carriage-return (CR) and line-feed (LF) character. One of these data files contains information such as the half-time, decay modes, and branching fractions of the radionuclides and is used by DCFPAK to assemble the information on the decay chain for the parent radionuclide. The other nine data files contain dose coefficients corresponding to the nine exposure modes described earlier. A short description of each of the ten data files is given in the following.

\section{DFEXTINT.NDX}

This file is used by DCFPAK to assemble the information on the decay chain for the parent. The file contains 839 lines, including a header line with such information as the record format and one line of information for each of the 838 radionuclides addressed by DCFPAK. The line of information for a radionuclide contains the name and half-life of the radionuclide, the key (line number) of the radionuclide record in all other files, and a summary of decay properties of each radionuclide. For example, the line of information for $\mathrm{Bi}-212$ gives the following information in the indicated order:

name of radionuclide (Bi-212);

half-life $(60.55 \mathrm{~m})$; decay modes ( $\mathrm{B}-\mathrm{A}$, indicating beta-minus and alpha); beginning line number for $\mathrm{Bi}-212$ in the inhalation dose coefficient file (2282); record number for $\mathrm{Bi}-212$ in the ingestion dose coefficient file (699); record number for $\mathrm{Bi}-212$ in each of the external dose coefficient files (690); record number in DFEXTINT.NDX of decay product Po-212 (504); branching fraction to Po-212 (6.4070E-01); record number in DFEXTINT.NDX of decay product Tl-208 (762); branching fraction to Tl-208 (3.5930E-01); total energy $(\mathrm{MeV})$ of emitted alpha particles (2.1741); total energy (MeV) of emitted beta particles ( 0.4720 ); total energy (MeV) of emitted photons ( 0.1855$)$; atomic weight (211.991271); date of creation of the radioactive decay data (05-May-77). 


\section{DFINHS.DAT}

This file is an expanded version of Table 2.1 of Federal Guidance Report No. 11. The file contains dose coefficients for the case of acute intake of a radionuclide by inhalation. The dose coefficients represent the dose over a 50-year period following intake ( $\mathrm{Sv} \mathrm{Bq}{ }^{-1}$ inhaled). Tabulated dose coefficients are for the default particle size $1 \mu \mathrm{m}$ (activity median aerodynamic diameter, or AMAD), except for a few cases in which particle size is not relevant (e.g., for vapors). As described below, the tabulated dose coefficients are used to generate dose coefficients for any particle size specified by the user.

For each radionuclide, inhalation dose coefficients are included for each of the lung clearance classes applied to that radionuclide in ICRP Publication 30 (1977, 1980, 1981, 1988). The three main clearance classes are "D", "W", and "Y", where Class $D$ refers to a clearance time of days, W to a clearance time of weeks, and $Y$ to a clearance time of years from the pulmonary region of the lung. The occasionally used Class " $V$ " refers to rapid clearance expected to occur for vapor forms of some elements. Three special clearance classes are used for carbon: "c", applied to labeled organic compounds, " $m$ ", applied to carbon monoxide, and " $\mathrm{d}$ ", applied to carbon dioxide; differences in the three classes are associated mainly with differences in the relative amounts of deposited carbon exhaled and absorbed to blood and in the biokinetics of carbon after absorption to blood.

For each radionuclide, one or more lines of data are included for each clearance class. The first line includes the name of the radionuclide, the clearance class, the gastrointestinal absorption fraction $\left(f_{1}\right.$ value) for material cleared to the gastrointestinal tract, a dose coefficient for each of the 23 tissues considered (Table 1), the effective dose equivalent, and the effective dose. For clearance class $\mathrm{D}, \mathrm{W}$, or $\mathrm{Y}$ (which apply to particulate matter), a second line of data provides the percentage of the dose coefficient for each organ that is attributable to deposition in the nasal-pharynx (N-P) and pulmonary $(\mathrm{P})$ regions of the respiratory tract. As described below, these percentages are used by DCFPAK to calculate dose coefficients for particle sizes other than $1 \mu \mathrm{m}$.

Dose coefficients for particle sizes other than $1 \mu \mathrm{m}$ can be derived from the tabulated data for $1 \mu \mathrm{m}$ as a result of the assumption in the underlying respiratory tract model (ICRP 1979) that the rate of clearance of material from the tract is independent of particle size. This assumption implies that the inhalation dose coefficient for a given radionuclide and clearance class is determined by the relative fractions of inhaled activity assigned to N-P, T-B, and P. Suppose, for example, that the user is considering inhalation of $\mathrm{Be}-10$ of Class $\mathrm{W}$ and particle size $0.2 \mu \mathrm{m}$. For this particle size, the deposition fractions for $\mathrm{N}-\mathrm{P}, \mathrm{T}-\mathrm{B}$, and $\mathrm{P}$ are $0.05,0.08$, and 0.5 , respectively (with the missing fraction, 0.37 , assumed to be promptly exhaled). For particle size $1 \mu \mathrm{m}$, the deposition fractions for $\mathrm{N}-\mathrm{P}, \mathrm{T}-\mathrm{B}$, and $\mathrm{P}$ are $0.30,0.08$, and 0.25 , respectively. According to the file DFINHS.DAT, for Be10 of Class W and particle size $1 \mu \mathrm{m}, 26 \%$ of the inhalation dose coefficient (DC) for breast, for example, is attributable to activity deposited in N-P and $41 \%$ is attributable to activity deposited in P. Therefore, $33 \%$ is attributable to activity deposited in T-B. The dose coefficient for the breast for $0.2 \mu \mathrm{m}$ would be 
$[(0.05 / 0.30) \times(0.26 \times D C)]+[(0.08 / 0.08) \times(0.33 \times D C)]$

$+[(0.5 / 0.25) \times(0.41 \times D C)]=1.1933 \times D C$,

i.e., 1.1933 times the dose coefficient for particle size $1 \mu \mathrm{m}$.

For a given particle size in the range $0.1-20 \mu \mathrm{m}$, deposition fractions for the regions $\mathrm{N}-\mathrm{P}, \mathrm{T}-\mathrm{B}$, and $P$ are based on the piecewise linear curves shown in Fig. 1 (after Fig. 5.1 of Part 1 of ICRP Publication 30 (1979)). For particle sizes less than $0.1 \mu \mathrm{m}$ or greater than $20 \mu \mathrm{m}$, DCFPAK assigns fractional depositions in these regions corresponding to a particle size of $0.1 \mu \mathrm{m}$ or $20 \mu \mathrm{m}$, respectively.

The esophagus was not depicted explicitly in the dosimetric human phantom used in ICRP Publication 30 and was not considered as a target organ in that document. However, the esophagus is addressed in DCFPAK because dose to the esophagus is considered in the calculation of the effective dose, E. Because internal dose calculations are not available for the esophagus, the thymus has been used as a surrogate. The esophagus was recently introduced into ORNL's human phantom and was considered explicitly in the external dose coefficients tabulated in Federal Guidance No. 12 and in DCFPAK.

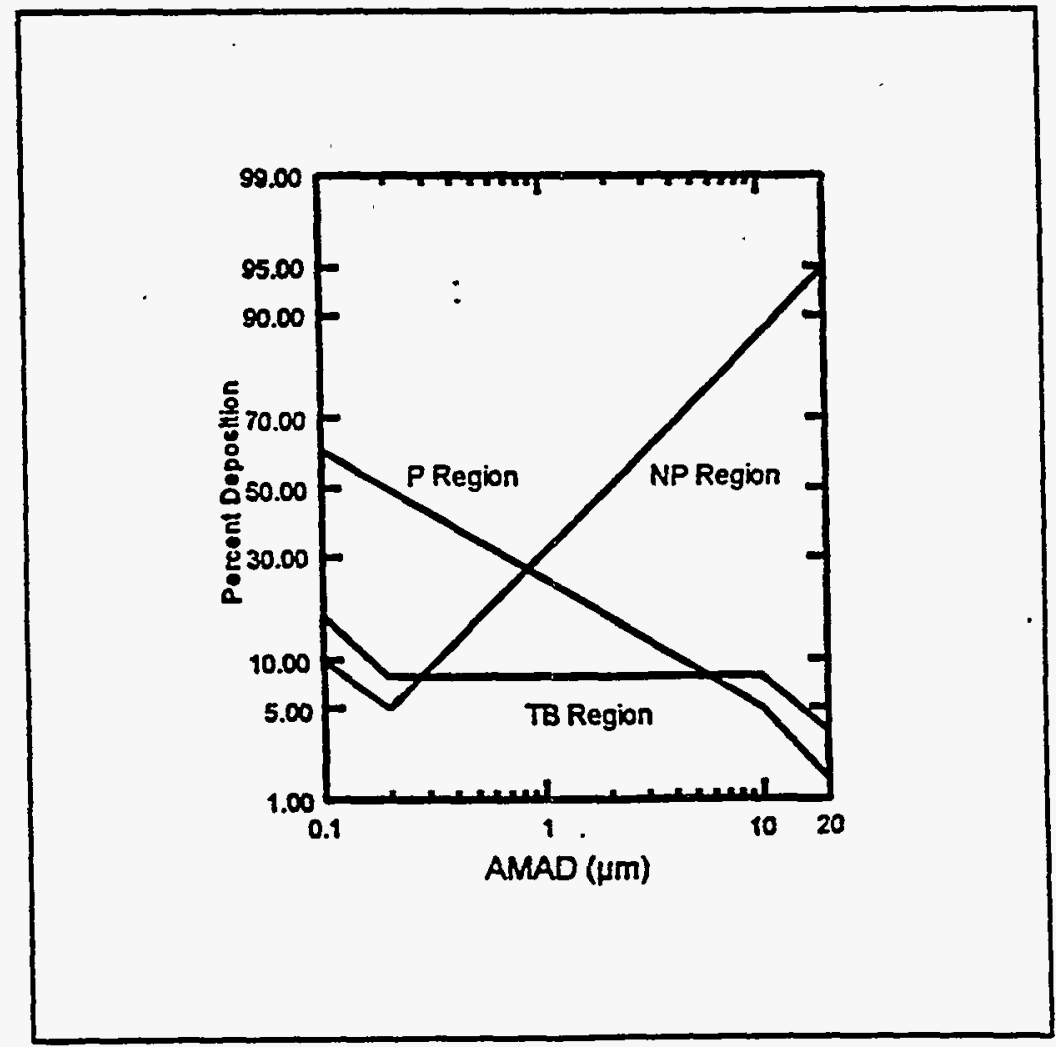

Figure 1 Deposition of particulate materials in the regions of the lung as a function of the activity median aerodynamic diameter (AMAD). 
For radionuclides addressed in Part 1 of ICRP Publication 30 (1979), an inhalation dose coefficient of 0.0 is given for brain. This is because the brain was not included in the human phantom used in the ICRP dosimetry until after the completion of Part 1 , and because there is no suitable surrogate for brain among the organs addressed.

\section{DFINGS.DAT}

This file is an expanded version of Table 2.2 of Federal Guidance Report No. 11. The file contains dose coefficients for the case of acute intake of a radionuclide by ingestion. The dose coefficients represent the dose over a 50-year period following acute intake ( $\mathrm{Sv} \mathrm{Bq}{ }^{-1}$ ingested).

With a few exceptions, one line of data is given for each radionuclide. That line includes the name of the radionuclide, the gastrointestinal absorption fraction ( $f_{1}$ value) for ingested activity, a dose coefficient for each of the 23 tissues considered (Table 1), the effective dose equivalent, and the effective dose. For a few radionuclides, one or more additional lines of dose coefficients are given, corresponding to alternate $f_{1}$ values used for certain forms of the radionuclide. For example, for radioisotopes of strontium, one line of dose coefficients corresponds to the $f_{1}$ value of 0.3 used for strontium compounds other than titanate and a second line gives dose coefficients for the $f_{1}$ value of 0.01 applied to strontium titanate.

For the reasons given in the discussion of inhalation dose coefficients, an ingestion dose coefficient of 0.0 for brain is given for each of the radionuclides addressed in Part 1 of ICRP Publication 30 (1979). Also, for reasons discussed above, ingestion dose coefficients for the thymus are applied to the esophagus.

\section{FGR12F31.DAT}

This file is an expanded version of Table III.1 of Federal Guidance Report No. 12. The file contains dose coefficients for the case of external exposure to the radionuclide in air (submersion). The units are dose per time-integrated air concentration ( $\mathrm{Sv}$ per Bq-s $\mathrm{L}^{-1}$ air).

One line of data is given for each radionuclide. That line contains the name of the radionuclide, a dose coefficient for each of the 23 tissues considered (Table 1), the effective dose equivalent, and the effective dose.

\section{FGR12F32.DAT}

This file is an expanded version of Table III.2 of Federal Guidance Report No. 12. The file contains dose coefficients for external exposure to the radionuclide in water ("immersion"), from swimming. The units are dose per time-integrated water concentration ( $\mathrm{Sv}$ per Bq-s L ${ }^{-1}$ water). 
One line of data is given for each radionuclide. That line contains the name of the radionuclide, a dose coefficient for each of the 23 tissues considered (Table 1), the effective dose equivalent, and the effective dose.

\section{FGR12F33.DAT}

This file is an expanded version of Table III.3 of Federal Guidance Report No. 12. The file contains dose coefficients for external exposure to the radionuclide on the ground surface. The units are dose per time-integrated surface concentration ( $\mathrm{Sv}$ per Bq-s $\mathrm{m}^{-2}$ ).

One line of data is given for each radionuclide. That line contains the name of the radionuclide, a dose coefficient for each of the 23 tissues considered (Table 1), the effective dose equivalent, and the effective dose.

\section{FGR12F34.DAT}

This file is an expanded version of Table III.4 of Federal Guidance Report No. 12. The file contains dose coefficients for external exposure to the radionuclide distributed to a depth of $1 \mathrm{~cm}$ in the soil. The units are dose per time-integrated concentration ( $\mathrm{Sv}$ per Bq-s $\mathrm{m}^{-3}$ ).

One line of data is given for each radionuclide. That line contains the name of the radionuclide, a dose coefficient for each of the 23 tissues considered (Table 1), the effective dose equivalent, and the effective dose.

\section{FGR12F35.DAT}

This file is an expanded version of Table III.5 of Federal Guidance Report No. 12. The file contains dose coefficients for external exposure to the radionuclide distributed to a depth of $5 \mathrm{~cm}$ in

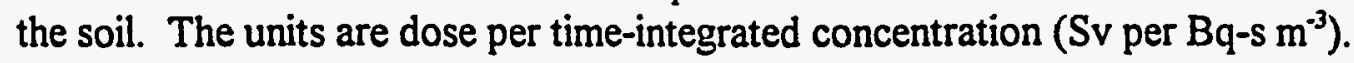

One line of data is given for each radionuclide. That line contains the name of the radionuclide, a dose coefficient for each of the 23 tissues considered (Table 1), the effective dose equivalent, and the effective dose.

\section{FGR12F36.DAT}

This file is an expanded version of Table III.6 of Federal Guidance Report No. 12. The file contains dose coefficients for external exposure to the radionuclide distributed to a depth of $15 \mathrm{~cm}$ in the soil. The units are dose per time-integrated concentration ( $\mathrm{Sv}$ per Bq-s m $\mathrm{m}^{-3}$ ).

One line of data is given for each radionuclide. That line contains the name of the radionuclide, a dose coefficient for each of the 23 tissues considered (Table 1), the effective dose equivalent, and the effective dose. 


\section{FGR12F37.DAT}

This file is an expanded version of Table III.7 of Federal Guidance Report No. 12. This file contains dose coefficients for external exposure to the radionuclide distributed to an infinite depth in the soil. The units are dose per time-integrated concentration $\left(\mathrm{Sv}\right.$ per $\left.\mathrm{Bq}-\mathrm{s} \mathrm{m}^{-3}\right)$.

One line of data is given for each radionuclide. That line contains the name of the radionuclide, a dose coefficient for each of the 23 tissues considered (Table 1), the effective dose equivalent, and the effective dose. 


\section{DCFPAK SOFTWARE}

The DCFPAK software files, written in FORTRAN 77, are described below. In the following descriptions, the code that calls DCFPAK's data access module will be referred to as the "user's driver code".

\section{DCFPAK.FOR}

This is the FORTRAN source code for the data access module that accesses the data files and returns the relevant information. It should be compiled on the user's system.

\section{PAKPARM.FOR}

This "include" file should be referenced by the user's driver code, along with the include file DCFPAK.CMN described below. The file contains the following two lines of FORTRAN code that determine the dimensioning of arrays in DCFPAK:

integer mspec, morg, mfact

parameter $($ mspec $=25$, morg $=25$, mfact $=9$ )

Here, MSPEC is the maximum length of the decay chain, MFACT is the maximum number of dose factors types, and MORG is the maximum number of organs in the dose factor file.

\section{DCFPAK.CMN}

This include file should be referenced by the user's driver code in any routines that access dosimetric information for the chain members. As noted above, PAKPARM.FOR should also be included in these routines. The file contains the common blocks that will contain the information extracted from the data files by DCFPAK:

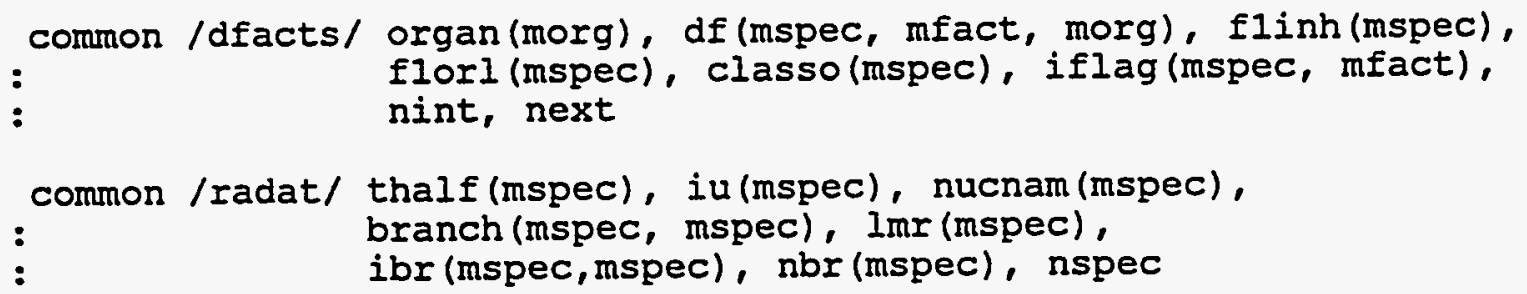

The common block DFACTS contains the dose coefficients. The variables in the dimension statements are defined in the include file PAKPARM.FOR. The definitions of the variables in this block are as follows:

ORGAN: A character(*9) array of organ names of maximum length MORG. The 23 organs addressed by DCFPAK are listed in Table 1. 
DF: A real variable array of dose coefficients by chain member, exposure pathway, and organ.

FIINH: Real variable array of gastrointestinal uptake $\left(f_{1}\right)$ values for inhalation, by chain member.

F1ORL: Real variable array of gastrointestinal uptake $\left(f_{1}\right)$ values for ingestion, by chain member.

CLASSO: A character $\left({ }^{*} 1\right)$ array of clearance class notation for the inhalation coefficients of the chain members.

IFLAG: An array of logical flags, by chain member and pathway, set to true in each case for which DCFPAK returns a coefficient in DF array.

NINT: Integer variable for the number of chain members that are used in the evaluation of inhalation and ingestion intakes (i.e., the length of the chain, or truncated chain, used in the calculation).

NEXT: Integer variable for the number of chain members that must be considered for external exposures.

The common block RADAT, shown above, contains information on the decay chain under consideration. The definitions of the variables in this block are as follows:

THALF: A character $(* 8)$ array of the half-life of each chain member.

IU: A character( $\left.{ }^{*} 2\right)$ array of the units of the half-life of each chain member.

NUCNAM: A character(*7) array of the names of the chain members.

BRANCH: A two-dimensional real array (upper triangular matrix) of branching fractions, with an entry representing the fraction of the decays of the ith chain member forming the $j$ th chain member.

LMR: A double precision array of decay constants $\left(\mathrm{d}^{-1}\right)$ for the chain members.

NBR: An integer array containing the number of chain members that decay directly to the specific chain member.

IBR: An integer array containing the indices of the chain members which form the specific chain member. For example, (ibr(I, ispec), $I=1, n b r(i s p e c)$ ) are the indices of the precursor of member ispec. 
NSPEC: The length of the decay chain.

\section{BATCH.CMN}

This include file should be referenced by the user's driver code. The file contains two lines of code, including a common block with a logical flag that determines whether DCFPAK is operated in an interactive or a non-interactive mode:

logical dbatch

common/dcalbat/dbatch

The DBATCH logical variable inserted into the user's code should be set to "false" for the interactive mode and "true" for the non-interactive mode. Setting the DBATCH logical variable to "true" eliminates output to the screen that occurs in the interactive mode.

\section{IOLIST.CMN}

This include file should reside in the same directory as the file DCFPAK.FOR when the latter is compiled. This four-line file contains the unit designations for the IO used by DCFPAK:

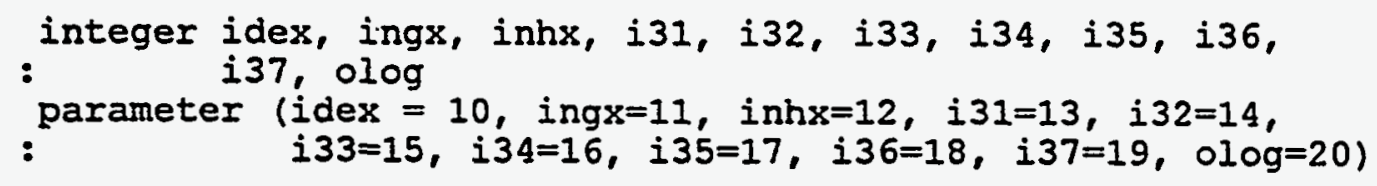

The unit designations in IOLIST.CMN must be reserved for DCFPAK input and output; that is, they must differ from unit designations in the user's driver code. In addition to these units, DCFPAK uses unit 40.

\section{DCFPAK.INI}

This "initialization" file should be placed in the directory where the software is run. The file informs DCFPAK of the location of all data files and their record lengths. This file enables the user to place DCFPAK's data files in a directory distinct from that used for code output. The following is a sample version of DCFPAK.INI. The real version should show the actual path to each file indicated in the second field:

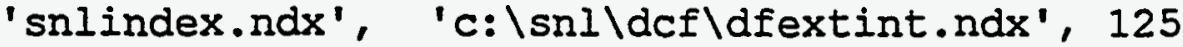

'ingestsf.dfs', 'c:\snlldcfldfings.dat' , 240

'inhalesf.dfs', 'c:\snl\dcfldfinhs.dat', 232

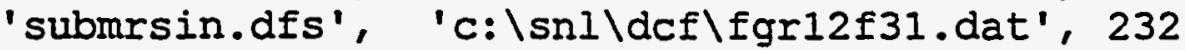

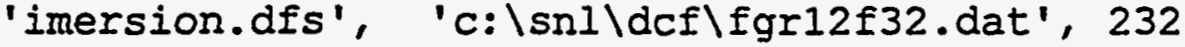

'grsurf00.dfs', 'c: \snl\dcflfgr12f33.dat', 232

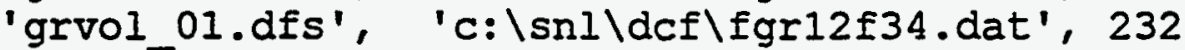




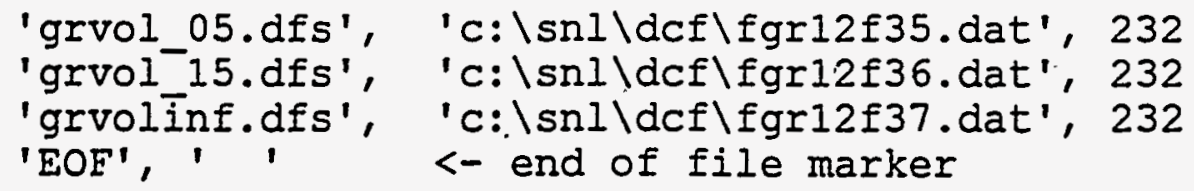

The first field is the file name within DCFPAK, and the second field is the full name of the file, including its path. For example, the record

$$
\text { 'snlindex.ndx', 'snlldcfldfextint.ndx' }
$$

indicates that the file 'snlindex.ndx' is to be read as snlldcfldfextint.ndx, where "snlldef" is the path to the directory containing the file. The user should not edit the first field and should limit the path in the second field so that the field length does not exceed 64 . The third field is the integer value for the record length for the file to be used in the FORTRAN open statement.

Not all FORTRAN compilers define the record length in the same manner when opening direct access files. DCFPAK was developed using the Microsoft FORTRAN compiler, which uses the physical length of the record less 2 bytes corresponding to the carriage return (CR)- line feed (LF) that separate formatted direct records. The integer values in the third field above specify the RECL parameter for the Microsoft compiler. Some other compilers (e.g, Lahey) specify RECL as the length of the physical record, which includes the 2 bytes associated with the CR-LF. For such compilers, DCFPAK.INI should be edited to increase the integer values indicated above by 2 . For additional information, see the comments included in the files after the EOF marker.

\section{READEM.FOR}

This is the source code for a sample driver code that accesses DCFPAK and returns to the screen the decay chain and dose coefficients for the chain members, based on the user's input. This code is included for illustrative purposes.

\section{READEM.EXE}

This is the executable file for READEM.FOR. 


\section{CONNECTING DCFPAK TO THE USER'S DRIVER CODE}

The DCFPAK data files and software are distributed in a self-extracting ZIP file named SNL.EXE. After extraction, the DCFPAK package can be connected to the user's driver code as follows:

Step 1: Copy the file snl.exe into a directory, preferably the directory that contains the user's driver code.

Step 2: Type "snl" and hit the "Enter" key. This extracts all data and software files. The data files may be moved to another directory. The new address of the data files must be specified in the file DCFPAK.INI.

Step 3: In the user's driver code, insert include statements referencing the include files PAKPARM.FOR, DCFPAK.CMN, BATCH.CMN, and IOLIST.CMN as illustrated in the sample driver source code, READEM.FOR.

Step 4: Insert the following "call" into the user's drive code, as illustrated in READEM.FOR:

CALL DOSECOF (NUKE, F1ORAL, CLASS, FIINHI, AMAD, IPATH)

to obtain the information for radionuclide NUKE. The dose conversion factor information is returned in the common blocks discussed above. The variables NUKE and IPATH must be specified. If the other variables are not specified, default values are supplied by DCFPAK. If the library has multiple sets of dose coefficients (e.g., corresponding to different fl values for a radionuclide), then DCFPAK will assign the most conservative set of coefficients. To obtain a particular set of coefficients one can specify the fl values for inhalation and ingestion as well as the clearance class for inhalation. These variables are described in the following section. Illustrative read statements are given in the sample driver source code, READEM.FOR.

Step 5: As discussed in the following section and illustrated in READEM.FOR, insert data input statements into the user's driver code to define the radionuclide (NUKE), the operative exposure pathway (IPATH), and optional input if used.

Step 6: Insert the calls "CALL OPENEM" and "CALL CLOSEM" into the user's driver code in appropriate places for opening and closing the data files, respectively, as illustrated in READEM.FOR.

Step 7: Compile DCFPAK.FOR and the user's driver code, and link the resulting object files. 


\section{INPUT TO THE USER'S DRIVER CODE}

Prior to calling the routine DOSECOF, a call should be made to the routine NUKEOK to determine if the nuclide of interest is contained in DCFPAK's data files. Thus, the user's code should include the following call:

\section{CALL NUKEOK(NUKE, OK)}

The variables are defined as:

NUKE: This character*7 variable represents the name of the radionuclide of interest. The naming convention for radionuclides follows standard practice (e.g., Co-60, Cs-137, Tc$99 \mathrm{~m})$. DCFPAK includes a character*7 function CHECK, which is called to place the nuclide name in the proper format.

OK: This is a logical variable which is set to true if the nuclide is present in the data files and is otherwise returned as false.

If the nuclide is present in the data files, then the user's driver code should access the data files through the call to DOSECOF described above:

\section{CALL DOSECOF (NUKE, F1ORAL, CLASS, FIINHI, AMAD, IPATH)}

The user's driver code should be set up so that the variables in this call are input variables, even though default values are supplied by DCFPAK for most of these variables. The variables are defined in the following.

NUKE: This variable was defined above.

F1ORAL: This is a real variable representing the value of the $f_{1}$ parameter (gastrointestinal uptake factor) associated with the ingested chemical form of the radionuclide. $f_{1}$ represents the fraction of the ingested material reaching blood in the absence of radioactive decay. For most radionuclides, a single $f_{1}$ value is applied to all chemical forms. However, for uranium, plutonium, and a few other elements, separate $f_{1}$ values are applied to relatively soluble and relatively insoluble compounds. If F1ORAL is set to zero, DCFPAK will return the ingestion coefficients for the chemical form that has the highest effective dose.

F1INHI: This is a real variable representing the gastrointestinal uptake factor for inhaled material that moves from the respiratory tract to the gastrointestinal tract. If F1INHI is set to zero, DCFPAK will assign the default value to F1INH1 associated with the radionuclide and the CLASS variable. 
CLASS: This is a character ${ }^{*} 1$ variable that specifies the clearance class for inhaled forms of the radionuclide. The dose coefficients are based on the lung model of ICRP Publication 30, and the variable has values of "D", "W", "Y", and "V". Class D refers to a clearance time of days, $W$ to a clearance time of weeks, and $Y$ to a clearance time of years. The less frequently used Class "V" refers to rapid clearance such as occurs for vapor forms of some elements. If CLASS is not supplied, DCFPAK will select the classification of the chemical form giving the highest effective dose.

AMAD: This is a real variable representing the activity median aerodynamic diameter of the aerosol. If unspecified, a default AMAD of $1 \mu \mathrm{m}$ is applied. If specified, DCFPAK calculates coefficients for the user's supplied AMAD. DCFPAK applies the value of AMAD to all chain members.

IPATH: This is a logical array (size 9) used to flag the type of dose coefficients that are of interest to the user. IPATH should be set to "true" to return tables of dose coefficients for all nine exposure models addressed by DCFPAK.

In addition to assembling the decay chain and the corresponding dose coefficients, DCFPAK can be used to compute the activities of the chain members as a function of time. The calculations assume a unit activity of the chain parent at time zero and no initial activity of other chain members. To make use of this capability, the following call should be inserted into the user's driver code after a call to DOSECOF:

\section{CALL BIRCH(ISPEC, T, A, AINT)}

The variables are defined as follows:

ISPEC: This input variable is the index number of the chain member of interest. ISPEC ranges from 1, the chain parent, to NSPEC, the last radioactive member of the chain.

T: This input variable is the time (d) for which the activity of chain member ISPEC is to be computed.

A: This output variable of the routine BIRCH is the activity of ISPEC at time $T$ assuming that one unit of activity was present at $\mathrm{T}=0$ for the parent and all daughter activities at that time were zero.

AINT: The output variable is the time integrated activity of ISPEC during the period of length $T$, assuming that one unit of activity was present at $T=0$ for the parent and all daughter activities were zero at $T=0$. The unit is days. The number of nuclear transformations of ISPEC per $\mathrm{Bq}$ of the parent during this period would be 8.64E04 $\times$ AINT. 
To produce activities for multiple chain members and/or multiple times, the call may be looped over ISPEC and/or $T$. For long decay chains and short times the computed values A and AINT may exhibit numerical noise for some chain members due to loss of significance in the computations. Although these artifacts can be seen when tabulating values as a function of time, they are typically of no dosimetric consequence. An extended precision version of this routine is available from the authors but was not used in DFCPAK because its computations are considerably slower than those of the present version.

Before any calls to BIRCH, the routine DOSECOF must be called to assemble the decay chain. The user should see READEM.FOR for an illustrative implementation.

The user should examine the source code READEM.FOR for an example of how the routines of DCFPAK should be called. 


\section{DEMONSTRATION CODE}

The READEM code included with this software package is an interactive code that can be used to examine available DCFPAK output. To run the code, type "readem" and press <Enter>. You will be prompted for the name of the radionuclide, the inhalation clearance class (a default is provided, as explained earlier), and the particle size (a default value of $1 \mu \mathrm{m}$ is provided). In this demonstration code, default values are used for the other variables in the call DOSECOF. After responding to the three prompts described above, you will be shown the half-lives, branching fractions, and cumulative alpha, beta, and gamma energies of each member of the (non-truncated) decay chain for that radionuclide. Press <Enter> to see inhalation dose coefficients for the parent radionuclide, press $<$ Enter> again to see inhalation dose coefficients for the next decay chain member in the truncated chain, and so forth. Repeatedly pressing <Enter> will take you through the dose coefficients for all members of the truncated chain, for all nine exposure modes addressed in DCFPAK.

Following the listing of the dose coefficients assembled for the chain, you will be asked to provide a time at which the activities of the chain members should be computed. If this computation is not desired, enter a time of zero; you will then be prompted to enter the next radionuclide. Entering the time (in days) will produce a tabulation of the activity and time-integrated activity for the chain members, as derived by the routine BIRCH. This computation is carried out only for the chain members that have been judged by DCFPAK to be significant in the dosimetry.

To exit READEM, press < Enter> when prompted for the next radionuclide. 


\section{INSTALLATION ON UNIX WORKSTATION}

The following additional steps allow a user to run READEM on a UNIX (SUN) workstation:

1. Convert all files extracted from SNL.EXE (with the exception of convert2unix, wdaf, and wdaf.f) from DOS format to UNIX format. A batch file named convert2unix is included as an example of the necessary unix commands to convert the files.

2. Rewrite the data files as direct access files since the direct access attribute is lost in conversion from DOS to UNIX. A short FORTRAN program named wdaf (write direct access files) is included as an example of how to rewrite the files to restore the direct access attribute.

3. Replace the extension "for" on the files readem.for, dcfpak.for, and pakparm.for with extension " $\mathrm{f}$ ".

4. Replace the variable "nargs" in readem.f with "iargc".

5. Replace "len_trim" with "Inblnk" in readem.f and dcfpak.f.

6. Compile the package as

f77 -03 -native -libmil -o readem readem.f dcfpak.f SLIB 


\section{REFERENCES}

Eckerman, K. F.; Ryman, J. C. Federal Guidance Report No. 12: External Exposure to Radionuclides in Air, Water, and Soil. EPA-402-R-93-081. Washington, DC: U.S. Environmental Protection Agency; Office of Radiation and Indoor Air; 1993.

Eckerman, K. F.; Wolbarst, A. B.; Richardson, A. C. B. Federal Guidance Report No. 11: Limiting Values of Radionuclide Intake and Air Concentration and Dose Conversion Factors for Inhalation, Submersion, and Ingestion. EPA -520/1-88-020. Washington, DC: U.S. Environmental Protection Agency, Office of Radiation Programs; 1988.

International Commission on Radiological Protection. Annals of the ICRP 1. ICRP Publication 26. Oxford: Pergamon Press; 1977.

International Commission on Radiological Protection. Annual limits on intake of radionuclides by workers based on the 1990 recommendations. ICRP Publication 60. Oxford: Pergamon Press; 1991.

International Commission on Radiological Protection. Limits for intakes by workers. ICRP Publication 30. Oxford: Pergamon Press; Part 1: 1979.

International Commission on Radiological Protection. Limits for intakes by workers. ICRP Publication 30. Oxford: Pergamon Press; Part 2: 1980.

International Commission on Radiological Protection. Limits for intakes by workers. ICRP Publication 30. Oxford: Pergamon Press; Part 3: 1981.

International Commission on Radiological Protection. Limits for intakes by workers. ICRP Publication 30. Oxford: Pergamon Press; Part 4: 1988. 
APPENDIX A: Source Code Listings 


\section{A1. INCLUDE FIIES}

\section{INCLUDE FILE PAKPARM.FOR}

* The following parameter values give the maximium dimensions of the

arrays in the dose factor package. To increase the problem size edit this flle and recompile all routines.

mspec is the maximium number of nuclides in a chain

morg is the maxium number of organs in the dose factor file.

integer mspec, morg, mfact

parameter (mspec -25 , morg -25 , mfact -9 )

\section{INCLUDE EILE DCERAK.CMN}

\section{character*9 organ \\ character*8 thalf \\ character $* 7$ nucnam \\ character*1 classo}

real branch

double precision $1 \mathrm{mr}$

c Integer*4 $1 b r$, nbr, nint, next

logical iflag

common /dfacts/ organ (morg), df(mspec, mfact, morg), f1inh (mspec), : florl (mspeci, classo (mspec), Iflag (mspec, mfact), nint, next

common /radat/ thalf (mspec), lu (mspec), nucnam(mspec), : branch (mspec, mspec), Imr (mspec). Ibr (mspec, mspec),

\section{INCLUDE FILE BATCH.CMN}

\section{* SNL batch flag}

$\log 1$ cal dbatch
common/dcalbat/dbatch

\section{INCLUDE FILE IOLIST.CMN}

Integer 1dex, Ingx, Inhx, 131, 132, 133, 131, 135, 136, : 137, olog

133 $=10$, ingx=11, inhx-12, 131-13, 132m11,

\section{A2. PROGRAM DCEPAK. FOR}

DCFPAK: Dose Coefflclent File Package ORNL/TM-13347

K.F. Eckerman and R.F. Leggett

Oak Ridge National Laboratory

The following is DCFPAK's source code. The routines are presented in alphabetical order by function; the order 1s:
1. computational subroutines,
2. screen routines,
3. functions routines.

The code was written In EORTRAN 77 using the Micrsoft EORTRAN Compiler 5.1. Microsoft's LEN_TRIM function has been used, however at the end of the listing is a replacement function that can be activated by uncommenting the statements.

K.F. Eckerman July 1, 1996.

Routine call tree for DCFPAK

DOSECOE Entry polnt to DCFPAK

CHAIN

BIRCH Can call after DOSECOF

BATMAN

EXPE1

EXPEUN

EXPFUN

cLs

ERWARD

IBINRY 


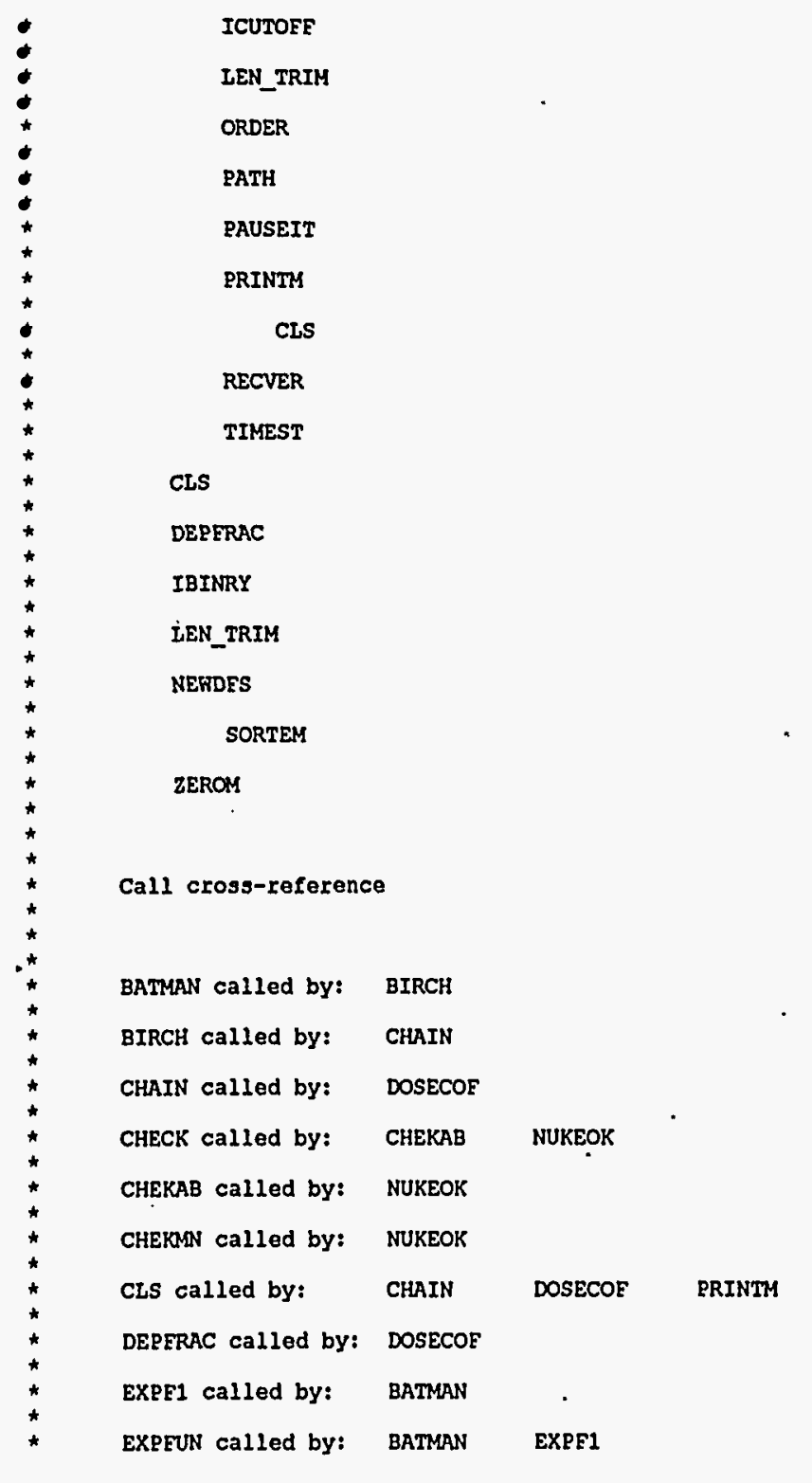

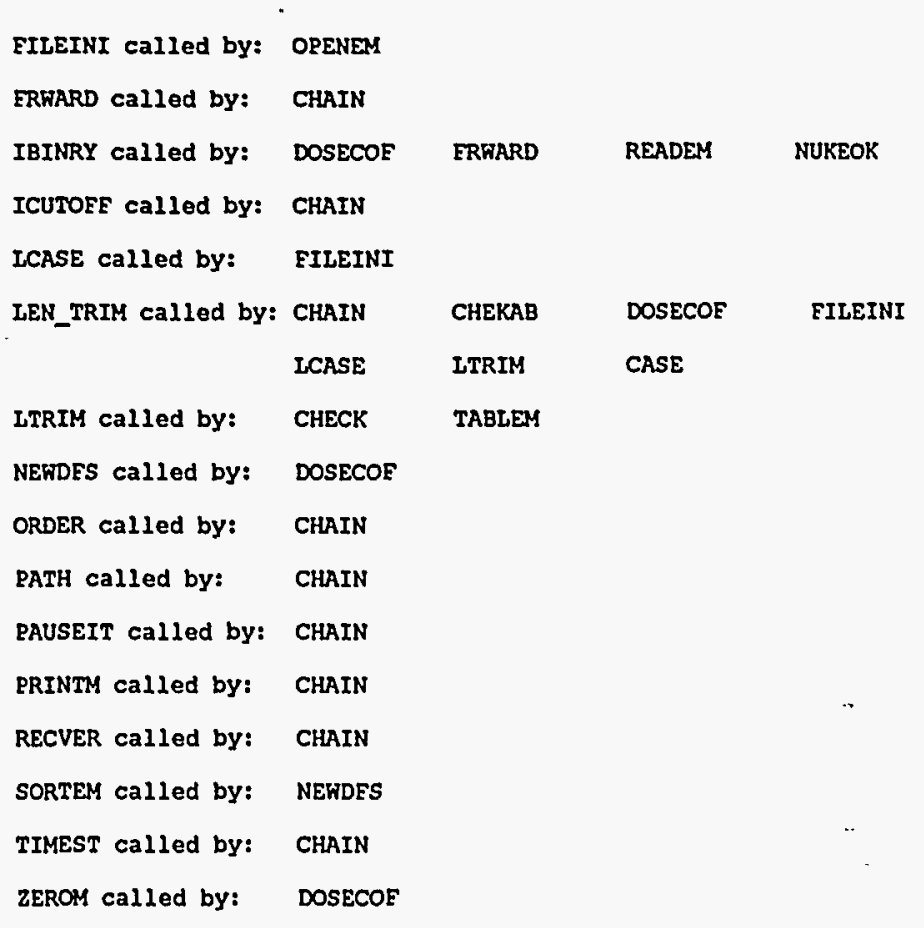

The user's code should call the following routines OPENEM -- to open the data files 
data organ /'Adrenals '', 'Bld Wall ', 'B Surface', 'Braln

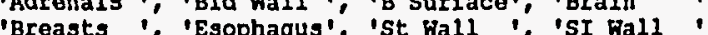
'ULI Wall ', 'LLI Fall ', 'KIdneys ', 'Liver 'Lungs '', 'Muscle ', 'Ovaries ' ', 'Pancreas ' 'R Marrow ', 'Skin ', 'Spleen ', 'Testes ', 'Thymus ', Thyroid ', 'Uterus ', 'H sub E ',

end

subroutine batman (b0, zk, zkt, an1, an2, $t, n$

Include 'pakparm.for'

call varlables.

double precision bo, zk, zkt, an1, an2, zero

integer $n$

dimension bo (mspec), zkt(mspec), zk (mspec)

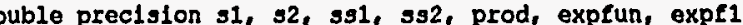

parameter (zero-0.0d0)

an1 - zero

an2 - zero

do $501-1$, n

f (bo(1). ne. zero) the

s1 = zero

$s 2$ - zero

$s s 1=$ zero

do $10 \mathrm{j}=1$,

prod $=2 k t(n)$

$z k(n) \cdot z k(j) / z k t(1)$

30 continue

If (prod .1t. zero) then

$s 1-s 1+$ dabs (prod) - expfun (-zkt (j) - dble(t)

else

$s 2=s 2+\operatorname{prod} \cdot \operatorname{expfun}(-z k t(j) \cdot \mathrm{dble}(t))$
$s s 2=\mathbf{s s 2}+\operatorname{prod} \cdot \operatorname{expf} 1(2 k t(j), \mathrm{dble}(t))$

40 continue

$\star$

only positive values are retained; negatives are zero

If (s2.gt. s1) an1 $=$ an1 + bo(1) (s2 - s1)

if (ss2 .gt. ss1) an2 $=$ an2 + bo 11 )

50 continue

return end

subroutine b1rch(imem, $t, r \times 1, x \times 2)$

nclude 'pakparm. for'

Include 'dcfpak.cmi

Integer mpath, max

common/calcul/ max (mspec), mpath (mspec, mspec)

call varlables.

local varlables.

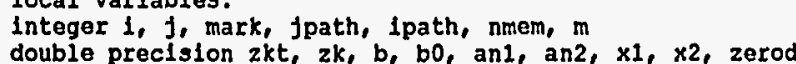

dimension b(mspec), bo (mspec), zkt (mspec), $2 k$ (mspec), mark (mspec) Jpath (mspec), Ipath (mspec)

Trace the pathway backwards from Imem to decide which elements of the Mpath matrix to choose.

\section{$r \times 1=2 e r 0$}

rX2 - zero

$x 1$ - zerod

$x 2$ zerod

do 301 - 1, nspec

$\operatorname{mark}(1)-1$

30 continue

31 nmem -1

fpath(1) - Imen

If (max(1mem) .eq. 0$)$ goto 35

33 Imem - mpath (mark (1mem), Imem) nmem - nmem +1

jpath (nmem) -1 mem 0 goto 33

35 do 10 (max (1mem)

ipath (1) - jpath (nmem - 1 + 1

40 continue

Imem - Ipath (nmem)

do $501=1$, nmem

bo(1) - b(1path(1))

2kt(1) - 1mr(1path(1)

If (1.1t. nmem) then

$2 k(1)$ - dble(branch (1path (1), 1path $(1+1))$ - zkt(1)

else

$2 k(1)-2 k t(1)$

call batman (bo, zk, zkt, an1, an2, $t$, nmem)

$x_{1}=x_{1}+$ an 1

60 do $801-1$ amen

b(1path (1)) - zerod 


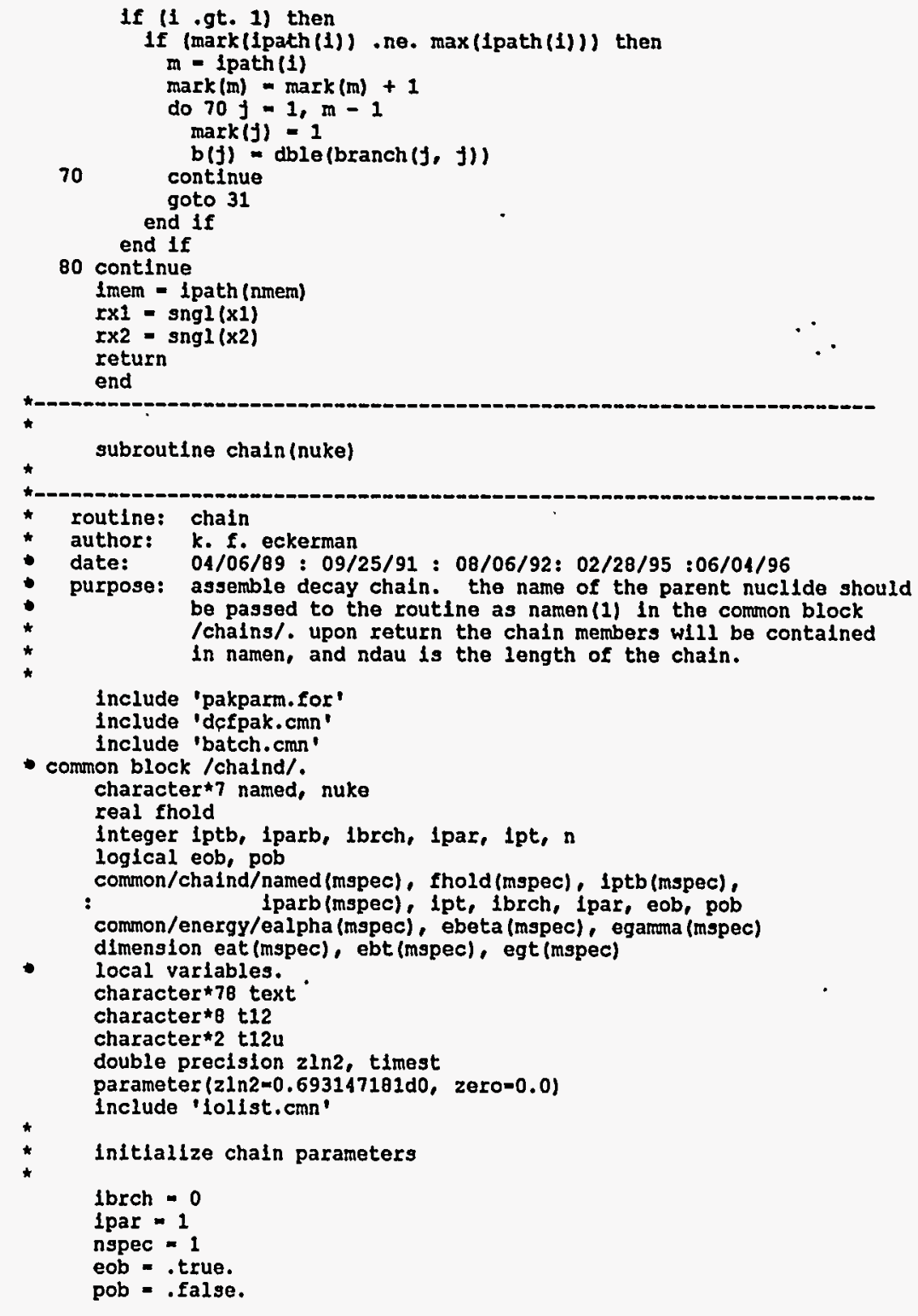

\section{nucnam(1) - nuke \\ do 11 do 10 , mspec}

10 branch $(1, j)$ - zero

10 continue

- assign one unit of activity to the parent, rest are zero

$$
\begin{aligned}
& \operatorname{branch}(1,1)=1.0 \\
& \operatorname{lm}(1)=0.0 \mathrm{~d} 0
\end{aligned}
$$

20 call frward

if (nspec .le. 0) then

1 - len_trim(nucnam(1))

write( $*^{-} \cdot(1 x,(a), "$ is not in data basel $" 1)$ ") nucnam(1) (:1) return

endif

call recver

if (.not. eob) goto 20

if (pob) nall - 1

do $1001-1$, napec

If (nucnam(1) $(: 2)$.eq. 'Sf') then

$\operatorname{lmr}(1)-0.0 \mathrm{DO}$

$1 \mathrm{mr}(1)=2 \ln 2 /$ timest (thalf(1), $1 u(1))$

100 continus

continue

t12 - thalf (1)

text - nucnam(1) (: len trim(nucnam(1)) // "Decay Cha1n:"

"Dranching"

text - text(:len_trim(text)) // - Fractions

1f (.not. dbatch) then

write $\left(*^{*}\right)$ text(:len_trim(text))

write $\left\{0 \log _{1}\right.$ '(/a) $) \cdot \operatorname{text}\left(: \operatorname{len}_{\text {trim(text) }}\right)$

ate parnto

.gt. 5) call cls

end if path

timess - 36525.0

- 1f (.not. dbatch) then

text - : Activity, Transformations, c cumulative Energles (MeV) :t $100 y^{\prime}$

write $(*, *)$ nucnam(1) (:len_trim(nucnam(1))), text $(:$ len_trim(text))

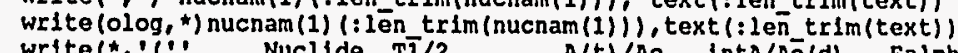
writel*, $(")$ Nuclide $\overline{1} / 2$ A(t)/Ao intA/Ao(d) Ealph -a Ebeta Egamma'")

writelolog, ' $"$ Nuclide $T 1 / 2$. A(t)/AO IntA/Ao(d) Ea ipha $1 f$

ea $=$ zero
eb $=$ zero

$\mathrm{eb}=$ zero
$\mathrm{eg}=$ zero 
do 50 ispec -1 , nspec

(

call birch (15pec, timess, $r \times 1, r \times 2$ )

ea - ea + rx2: ealpha (1spec)

eg = eg + rx2 - egamma(19pec)

16 (.not. dbatch) then

write(*, (11, 1x, a7, 1x, a8, a2, 1p2d12.5, 3e9.2)") 1spec, write (olog, $(14,1 x$, a7, $1 x$, ab, a2, 1p2d12.5, 3e9.2)' 1spec,

: nucnam(1spec), thalf(1spec), lu(1spec), rx1, rx2, ea, eb, eg

*

$$
\begin{aligned}
& \text { eat (1spec) }=\text { ea } \\
& \text { ebt (1spec) }=\text { eb } \\
& \text { egt (1spec) }=\text { eg }
\end{aligned}
$$

50 continue

if (nucnam (nspec) (:2) .eq. 'Sf') then nint - Leutoff (eat, ebt, egt, 0 , nspec-1)

next

- 1cutoff (eat, ebt, egt, 1, nspec-1)

nint - Icutoff (eat, ebt, egt, o, nspec) next

if (nspec

nint $=i$

next

end if

if (.not. dbatch) call pauselt

do 70 jspec -2 , nspec

$n=0$

do 60 1spec -1 , nspec

If (nucnam (1spec) (:2) isfi) goto 60

if (branch (ispec, ispec). gt. 1.0E-6) then

$$
n=n+1
$$

$1 b r(n$, Jspec $)$ - Ispec

60 continue

continue

-

70 continue

return$$
\text { : }
$$

subroutine chekab( nuke)

\section{routine: chekab}

author: M. Cristy

purpose: If nuclide not found, checks whether "a" \& "b" 1somers exist

character $\star 7$ nuke, nukeab, nukea, nukeb, check

character $\star 7$ nuke, nukeab,
character $\star 6$ thalf1, thaif2 paramater (nnuke - 9)

dimension nukeab (nnuke), nukea (nnuke), nukeb (nnuke),

: thalf1 (nnuke), thalf2 (nnuke)

'Np-236 / Eu-150 ' ' In-110' 'Ir-186 ', 'Nb-89 '',

data nukea /'Eu-150a', 'In-110a', 'Ir-186a', 'Nb-89a'.

.'Np-236a', 'Re-162a', 'Sb-120a', 'Sb-128a', 'Ta-178a'/'

data nukeb/'Eu-150b', 'In-110b', 'Ir-186b', 'Nb-89b',

- 'Np-236b', 'Re-182b', 'Sb-120b', 'Sb-128b', 'Ta-178b'/

data thalf1/'12.62h', '69.1m', '15.8h', '66m', '115E3y',

$112.7 \mathrm{~h}^{\prime}, 15.89 \mathrm{~m}^{\prime}, 10.4 \mathrm{~m}^{\prime}, 19.31 \mathrm{~m}^{\prime} /$

data thalf2/'34.2y', $4.9 \mathrm{~h}$ ', '1.75h', '122m', '22.5h',

64.0h', 5.76d',

do $101-1$, nnuke

If (nuke .eq. nukeab(1)) then

100 write (

nuclide or <Enter> to quit) $\rightarrow$ '

read $(*,+(b n, 27)$ ) $)$ nuke

nuke - check (nuke)

if (Ien_trim(nuke) .eq. o) stop

return

10 continue

return

9110 format ( $4 x$, 'Nuclide ', a, 'has 2 1somers: '/20x, $a$, ' with halfllfe ', a

- /16x, and $a_{\text {, }}$ with halflife,$a / 4 x_{\text {, }}$

-Re-input entire name with appropriate "a" or "b" designation',/ end

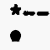

-

subroutine chekmn ( nuke)

routine: chekmn

date: $05 / 05 / 93$, rev1sed $8 / 16 / 93$

purpose: if " $\mathrm{m}^{\mathrm{n}}$ 1somer $1 \mathrm{~s}$ requested, checks whether " $\mathrm{n}$ " 1somer also exists.

character*7 nuke, nukem, nuken

character $* 6$ thalfm, thalf

character 1 meta

dimension nukem(3), nuken(3), thalfm(3), thalfn(3)

data nukem /'Ir-190m', 'Sb-124m', 'Tb-156m'/

data nuken / Ir-190n', 'Sb-124n', 'Tb-156n'/

data thalfm/'1.2h', '93'', '24.4h'/
data thalfn/3.2h', '20.2m', $5.0 \mathrm{~h} / /$

data nnuke $/ 3 /$

do $201-1$, nnuke

if (nuke. eq. nukem(1)) then

10 Write(*, g110) nuke, nukem(1), thalfm(1), nuken(1), thalen(1) write (* , givo) nuken

read $(*, \cdot$ (a1) $)$ meta

meta.eq. ' $M$ ') then

elself (mota.eq.' $n$ ' .or. meta.eq. ' $N$ ') then

nuke - nuke(1:6) $i{ }^{\circ}$ ' 


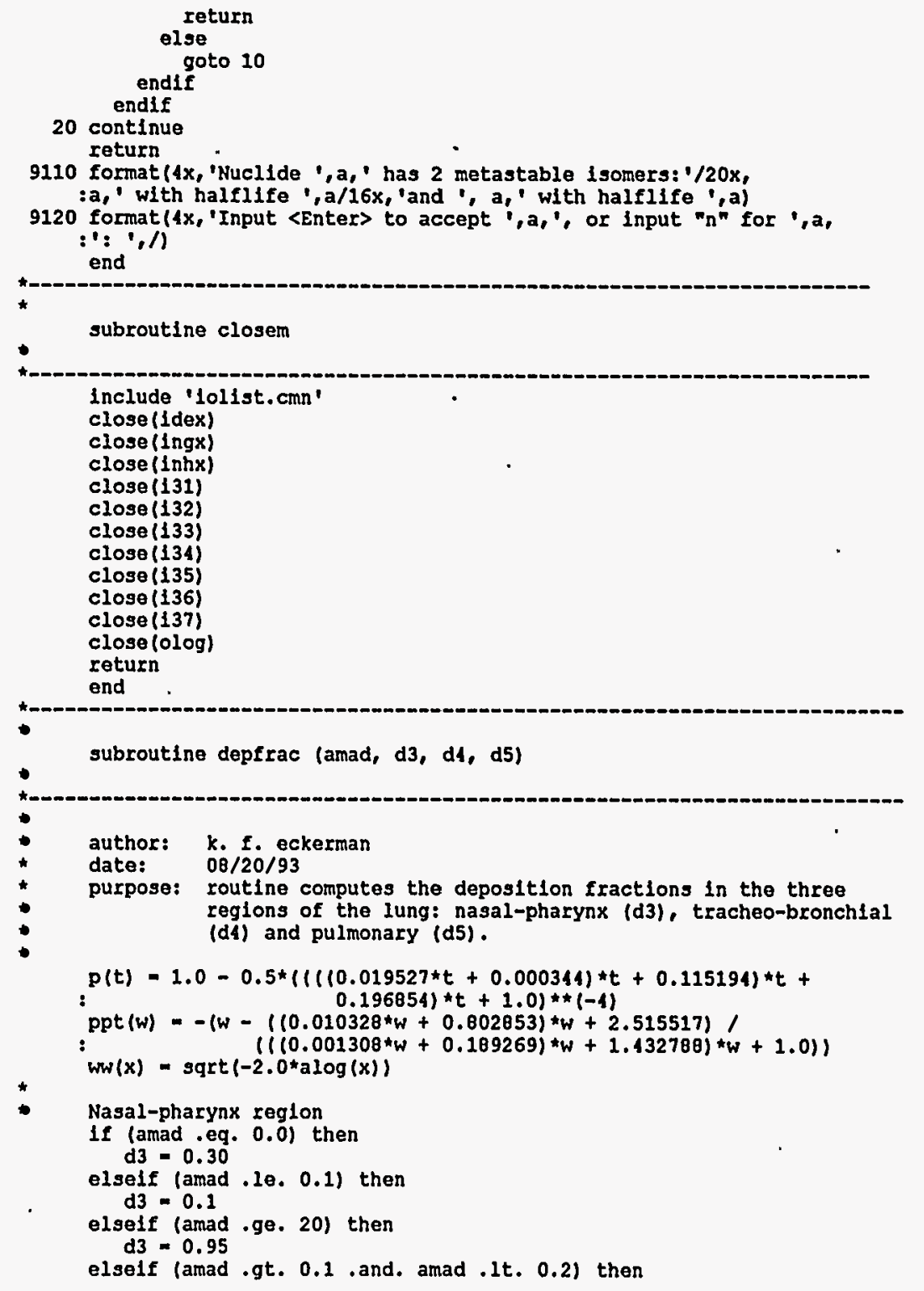

$40=-0.698970$

u1 $=-1.00$

to $=$ ppt (ww (0.05)

$t-t 0+(t 1-t 0) /(u 1-u 0) *(a \log 10(a m a d)-u 0)$ if $(t . g e$. 0.0$)$ then $d 3=p(t)$

else

$$
d 3-1.0-p(-t)
$$$$
\text { end if }
$$

0.2 .and. amad .1e. 10.0$)$ then

u1 $=-0.698970$

to $=\operatorname{ppt}\left(\mathrm{ww}_{1}(0.05)\right)$

$t 1=\operatorname{ppt}(\mathrm{ww}(0.5))$

$/(u 1-u 0) \cdot(a \log 10(a m a d)-40)$

if $(t . g e .0 .0)$ then

$d 3-p(t)$

else

$d 3-1.0-p(-t)$

elself (amad .gt. 10.0 .and. amad .1t. 20.0) then

uo $=1.0$

uI $=1.301030$

tI $=\operatorname{ppt}($ pw $(0.875))$

$t=t_{0}+(t 1$ - to) $/(u 1$ - u0) $(a \log 10($ amad $)-u 0)$

$1 f$ ( $t$.ge, 0.0$)$ then

$d 3=1.0-p(-t)$

end 1

- TB region

If lamad.eq. 0.0 ) then

d4 -0.08

elself (amad .1e. 0.1) the

d4 -0.17

20) then

d4 $=0.035$

elseif (amad .gt. 0.1 .and. amad .1t. 0.2 ) then

uo $=-0.698$

u1 $=-1.0$

t1 $=$ ppt (ww $(0.08))$

$t-t 0+(t 1-t 0) /(u 1-u 0) *(a \log 10(a m a d)-u 0)$

if ( $t . g e .0 .0)$ then

di $=p(t)$

else$$
\text { dite }
$$

$1.0-p(-t)$

lseif lamad

d4 $=0.08$

u0 $=1.0$

uo $=1.0$
uI $=1.30130$

$u 1=1.30130$
to $=\operatorname{ppt}(\mathrm{ww}(0.08))$ 
t1 - ppt (ww $(0,035)\}$

$t=t 0+(t 1-t 0) /(u 1-u 0)+(a \log 10$ (amad) - u0) if $(t) \cdot g e .0 .01$

else

$$
\text { d4. }
$$$$
\text { end if }
$$

* Pulmonary region

if (amad .eq. 0.0 ) the

d5 $=0.25$

elself (amad .1e. 0.1) then

d5 $=0.6150$

seif (amad.ge. 20.0) then

d5 $=0.015$

self (amad .gt. 0.1 .and. amad..1t. 0.2) then

$40=-0.6989$

to $=-1.00$

t1 = ppt(ww $(0.5))$

$t=t 0+(t 1-t 0) /(u 1-u 0)+(a) \log 10(a m a d)-u 0)$
if $(t-g e .0 .0)$ then

$$
\text { if }(t=g e \cdot 0
$$

else

$$
d 5=1.0-p(-t)
$$

0.2 . and. amad $.10,10.0)$ then$$
\mathrm{uO}=-0.698970
$$

U1 $=1.0$

to $=\operatorname{ppt}(\mathrm{ww}(0.5))$

t1 $=$ ppt (nw $(0.05))$

$t=t 0+(t 1-t 0) /(u 1-u 0) \cdot(a \log 10(a m a d)-u 0)$

if $(t .90 .0 .0)$ then

ds $=\mathrm{p}(\mathrm{t})$

$d 5=1.0-p(-t)$

elseif

seif (amad .gt. 10.0 .and. amad .1t. 20.0) then

$\begin{array}{ll}\text { u } & =1.0 \\ \mathrm{u} 1 & =1.301030\end{array}$

to $=\operatorname{ppt}($ ww $(0.05)$

t1 - ppt (ww $(0.015)$

$t=t 0+(t 1-t 0) /(u 1-u 0) *(a \log 10(a m a d)-u 0)$

If $(t . g e .0 .0)$ then

$d 5=1.0-p(-t)$

end if

50 continue

return subroutine dosecof (nuke, flo, class, fll, amad, Ipath)

\section{routine: dosecor}

author: K.f. eckerman

data: $05 / 20 / 96$

purpose: assemble the decay chain and extract the dose coefficlents for the chain members.

input variables

nuke parent of the chain in standard notation; e.g., Cg-137

f 1 value for oral intakes (defaults to highest effective

class will be of this class if possible or class with highes

11 effective dose.

f 1 value for inhalation. coefficlents are actualiy

plcked by class.

ipath (1) pathway loglcal flag of size 9. 1 Inhalation,

2 Ingestion, 3 submersion, 4 immergion, 5 ground surface,

$61 \mathrm{~cm}$ thick soil sample, $75 \mathrm{~cm}, 815 \mathrm{~cm}, 9$ infinite.

output

output is through the common blocks in the include file defpak.cmn

as shown below. : organ character $\star 9$ array of organ names.

df dose factors array by chain member, pathway, and organ

florl arry of 1 valueg for Ingegtion by chain member

classo array of ciass notatlon for inhalation by chaln member

iflag logical flags for dose factor by chain member and pathway

nint length of chain for Internal factors

next

common /radat/ thalf (mspec), iu (mspec), nucnam (mspec),

branch (mspec, mspec), Imr (mspec),

lbr (mspec, mspec), nbr (mspec), nspec

thalf half-life of chain members

units of the half-lives

branch branching fraction

Imr decay constant in $1 / d$

Ibr branch pointer

nor number of branches for chain members

include 'pakparm.for'

Include 'pakparm. for'

include crpak.cmn'

character 7 nuke, nuclide

character*1 clshid, class, cx 
integer 1pt, 1binry, 1dep

dimension $d x(4,25)$, fihld(4), clshld(4), 1dep (1, 25, 2), ifl(7)

if (111 131, 132, 133, 134, 135, 136, 137/

* zero out the dose factor array

call zerom

assemble the decay chain

call chain (nuke)

first do the external coefficients for the next chain members

do 20 ispec -1 , next

nuke = nucnam(1spec)

1f (ipt

read (idex, $(a 7, a 8, a 2, a 6,315) '$, recmlpt) nuke, $t, 1 x$, mode,

in

If (10x, ing, lex

do 10 ip $=3,9$

if (ipath(ip)) then

Iflag (ispec, ip)

ead(1f1(1p-2), $\cdot(27,1 \mathrm{p} 2509.0)$, rec $=10 x)$

end if nucllde, (df(1spec, ip, $f$ ), $j-1,25)$

10 continue
end if

end if

20 continue

now do the inhalation and ingestion coefficients

if (amad .ne. 1.0) call depfrac (amad, d3, d4, d5)

200 1spec $=1$, nint

nuke - nucnam(19pec)

read(1dex, $(27, a 8,22,26,315)$ ', rec-ipt) nuke, $t, 1 x$, mode, inh

ing, lex

c inhalation

if (1path(1).and. Inh .ne. 0) then

1 max -0

$\operatorname{dmax}=0.0$

1rec - Inh -

Iflag (1spec, 1) - .true.

do 50 itry $=1,4$

irec $=1$ irec +

read(1nhx, (a7, 1x, a1, e8.0, 25e9.0)', rec-irec, end-60)

$\begin{array}{ll}: & \text { nuclide, cx, flhld(1try) } \\ \text { (dx (1try, } f), j=1,25)\end{array}$

if we have a particulate factor then read the next record to get the contribution of the deposition in the $N P$ and $P$ region to the organ dose.

If (CX .eq. ' $D$ ' .or. CX .eq. ' $W$ ' .or. CX .eq. 'Y') then irec - irec + 1 read(1nhx, '(17x, 25(15, 11))', rec-1rec)

end if (1dep(1try, j, 1), 1dep(1try, J, 2), J $-1,25$ )

clshld(1try) $=\mathrm{cx}$

if (len_trim(nucllde) .gt. 0 .and. nuclide .ne. nuke) the goto 60

elself (len trim (nuclide).eq.o .or. nuclide.eq. nuke) then If (class .eq. clshld(itry) and. fil .eq. flhld(itry)) then

calculate the factors for this amad if $\longleftrightarrow 1 \mu \mathrm{m}$

if (amad.ne.1.0) call newdfs (1try,d3,d4,d5, dx, 1dep)

do $30 f-1,25$

df(1spec, $1, f)-d x(1$ try, $f)$

continue

flinh (1spec) - flhld(1try)

classo(lspec) - clshld(1try)

goto 80

elseif (class .eq. clshid(1try)) then

calculate the factors for thls amad if $<1 \mu \mathrm{m}$

If (amad.ne.1.0) call newdfs (1try,d3,d4,d5,dx, Idep)

do $10 \mathrm{j}-1,25$

df (1spec, $1, f)-d x(1$ try, f)

continue

flinh (1spec) - flhld(1try)

classo (1spec) - clshld(1try)

goto 80

(dx(1try, 25) .gt. dmax) then

$d \max =d x(1 t r y, 25)$

end if

end if

else

goto 60
end if

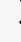

calculate the factors for this amad if $\leftrightarrow>1 \mu \mathrm{m}$

do $70 \mathrm{f}-1,25$

df $(1$ spec, $1, j)=d x(1 \max , j)$

continue
flinh (1spec) $=$ f1hld(1max) 


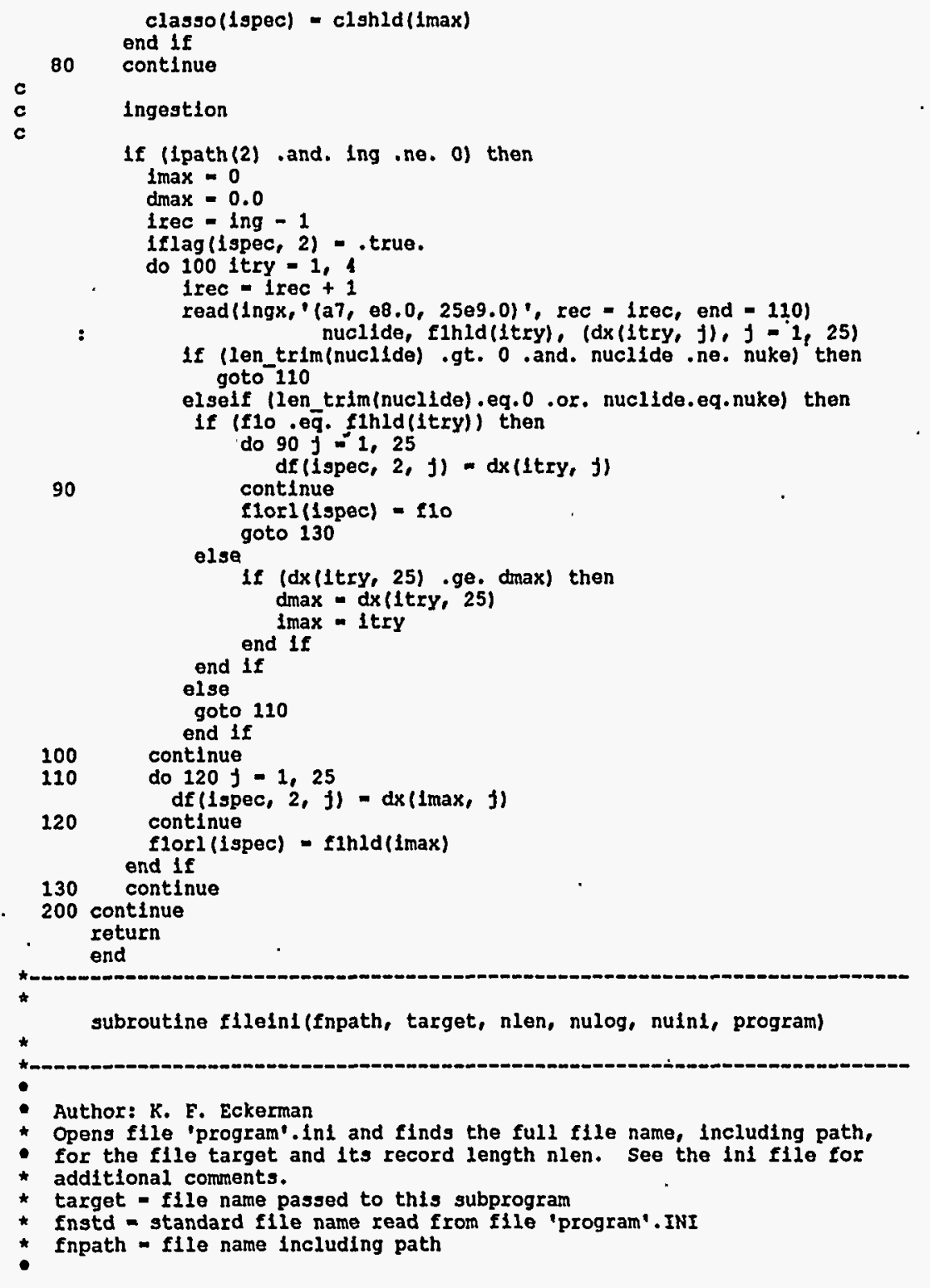

character*(*) fnpath, target, program

character 12 fnini, instd, lease

integer nulog

parameter (maxei1 - 10$)$

fnini - program(1:1en trim(program)) $/ 1 \cdot 1$. $^{\circ}$

Inquire (file - Inini, exist - test)

1f (. not. test) goto 20

open (nuIn1, Ellemfnin1, status-'old')

target $=1$ case (targot)

do $101: 1$, maxfil

read(nurni, *, end 1

If (fnstal:3) .eq. 'EOF') goto 15

10 continue

15 write(nuLog, 9110$)$ target (: len_trim(target)), fninl(: len_trim(fnin1) write (- 9110$)$ target (: len_trim(target)), fnIn1 (:Ien_trim(fnIn1) close (nuInl)

close (nuIni)
return

9110 formatl' **** EATAL ERROR in function ElleInl: Unable to find the :flle $, a, a^{\prime}, / 6 x$, 'check $, a, c^{\prime}$ for proper assignments $\left.* \star \star * 1\right)$

-

subroutine frward

routlne: frward

author: $k$. $f$. eckerman

purpose: read down a branch of a decay chain.

Include 'pakparm.for'

nclude 'dcfpak.cm

real fhold

integer 1ptb, Iparb, Ibrch, Ipar, Ipt

logical eob, pob

common/chalnd/named (mspec), fhold (mspec), 1ptb (mspec),

comon/energy/ealpha (mspec), ebeta (mspec), egamma (mspec)

- functions referenced.

integer ibinry

character $* 8 t$, mode

character $\star 7$ nuke, d1

character $* 2$ ix

include '1011st.cmn'

- get parent record. 
1f (ipar .eq. 1) then nuke - nucnam (1par) Ipt - Iblnry (nuke)

nept .eq. 0) nspec 0

endif

endif

10 if (lpt .1t. 999) then

read (idex,,$(27,28,22,26,315,3(14,011.0), 3 f 7.0, e 11,0,1 x, 29)$ ', $\vdots$ rec-ipt) nuke, $t$, $1 x$, mode, Inh, ing, lex, 1d1, 11 ,
Id2, f2, 1d3, f3, ea, eb, eg, amu, endsf else

Id1 $=$

$f 1=0.0$

$1 \mathrm{~d} 2=0$

$f 2=0.0$
$1 \mathrm{~d} 3=0$

$1 \mathrm{~d} 3=0$
$\mathrm{f3}=0.0$

nuxe $=$ 'sE'

ea $=0$.

eb $=0$

eg $=0$

txin

end $1 f$

Ids - 999 denotes "sf" whlch is not a daughter product, thus set the Ids to zero if "sf".

If (1d1 .eq. 999 .and. 1d2 .ne. 0) then

If $(1 \mathrm{~d} 3$.eq. 0$)$ then

fhld $=$ Id 1

$\mathrm{f1}-\mathrm{f2} 2$

$1 \mathrm{~d} 1=1 \mathrm{~d} 2$

f2 - fhld

else

fhld $=f 1$

lhld $=1 \mathrm{~d} 1$

Id $1=10$

$12=13$

$1 \mathrm{~d} 2=1 \mathrm{~d} 3$

f $3=$ thld

$1 \mathrm{~d} 3=\ln 1 \mathrm{~d}$

end

if (Id2 .eq. 999 .and. Id3 , ne. O) then

inld $=$ Id 2

fhld $=12$

$1 d 2=1 d$

$12=13$

$1 \mathrm{~d} 3=$ lhld

end if
If processing a branch then check to see if dl has already

a branch then check to seo if di has alroady terminate chain, 1.e.., chaln has converged.

if (pob) then

if (Id1 .gt. 0 .and. id1 .1t. 999) then

read (idex, 50, rec - Id 1 ) di

elseif (1d1. eq. 999) then

d1 - 'sf'

end if

do $15 \mathrm{y}=1$, nspec -

15 continue

$:$

have already handled this daughter: chain has converged. set end of chain and return.

16 branch (lpar, $f)-f 1$

(lpar) a nuk

ebeta(1par) = eb

egamma (1par) - eg

Iu(Lpar) $-1 x$

nspec $=$ nspec + 1

return

end $1 f$

- need to treat this chain member.

17 nucnam(1par) - nuke

thalf (lpar) $-t$

ealpha(1par) - ea

ebeta (lpar) = eb

egamma(1par) -

lu(lpar) $-1 x$

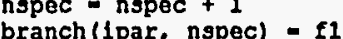

- no further daughters In chaln - set end of chain

If (Id1 ne. O) then

- further daughters, treat $1 d 1$ and check for possible branches. lpt $-1 \mathrm{~d} 1$

If (Id2 .ne. 0 ) then

set end of branch to false, increment branch counter, store pointer of parent, and record number of second or third daughter while following current chain. routine recver wlll direct recovery of branches.

eob - . false.

lbrch = Ibrch + 1 
fhold(1brch) $=f 2$

If (1d2 90.99 )

read(Idex, 50, rec - 1d2) named(1brch)

else

$$
\text { named(lbrch) }- \text { 'sf' }
$$$$
\text { end } 18
$$

third daughter, branch info held as above.

if (Id3 . ne. O) then

eob - . false.

1brch - Ibrch +

iptb (1brch) $=1 \mathrm{~d}$

(hold (1brch) $=13$

iparb(1brch) - ipar

(Id3 ine. 9g) then

- 1d3) named(1brch)

named(lbrch) - 'sf'

end

Ipar - nspec

If (1pt ne. 999) goto 10

endif

50 format $(a 7, a 8, a 2, a 8,17,15,16,14,3(14, e 11,0), f 7,0,2 f 6,0, a 10)$ end

subroutine newdfs $(1, d 3, d 4, d 5, d x$, Idep)

\section{routine: newdfs}

author: K.f. eckerman

date: compute hy

dimension $d x(4,25)$, 1dep $(4,25,2)$, dsort (12), 1sort (12)

data 1sort $/ 1,4,7,8,9,10,11,12,16,19,21,23 /$

* calculate the inhalation coefficlent for this new depositions

do $10 \mathrm{~J}=1,23$

$f=\mathrm{d} 3:$ lloat (1dep $(1, j, 1)) / 0.30+$

d5 * float (1dep(1, 3,2$)) / 0.25$

d4 (float (100-1dep(1,j,1) - 1dep (1, J,2)1)/0.08

10 continue

compute the effective dose equivalent (welghting factors of 1crp-26) and the effective dose (wolghting factors of lcrp-60) for the new depostions.

he $-0.25 \cdot \max (\mathrm{dx}(1,15), \mathrm{dx}(1,20))+0.15 \cdot \mathrm{dx}(1,5)+$ $\begin{array}{ll}: & 0.12: \mathrm{dx}(1,13)+0.12 \cdot \mathrm{dx}(1,17)+0.03 \cdot \mathrm{dx}(1,22)+\end{array}$ do $20 \mathrm{f}=1,12$

dsort (j) $-d x(1$, 1gort $(j))$

20 continue

call sortem (dsort, 12)

he $=$ he +0.06

dsort (B)i

$\max (d x(1,15), d x(1,20))+0.05 a d x(1,5)+$

$0.12 \cdot d x(1,10)+0.12 \cdot d x(1,17)+0.12 \cdot d x(1,13)+$

$0.12: \mathrm{dx}(1,7)+0.05: \mathrm{dx}(1,2)+0.05 \cdot \mathrm{dx}(1,12)+$

$0.01 * \mathrm{dx}(1,18)$

hr - (14. $d x(1,1)+1400, d x(1,4)+610, \cdot d x(1,8)+$ 310. $\mathrm{dx}(1,12)+20000$. $\mathrm{dx}(1,4)+14)+$ 180. $d x(1,19)+20 . \star d x(1,21)+60 . \cdot d x(1,23)) /$ 3074

$e+0.05 \cdot h$

$d x(1,21)=$ ho

return

\section{$:$}

subroutine openem

routine: openem

author: k.f. ockerman

date: $10 / 23 / 93$

purpose: open Index and dose coefficient files.

character $* 61$ fpath

character $\star 12$ target

character $\star 8$ prog

open (olog, flle - 'df read. $\log$ ')

prog - 'dcfpak'

target = 'snlfndex, $n d x$

cali fileini(fpath, target, nlen, olog, 11, prog) open (1dex, file-fpath, access-'dírect', recl-nlen, form: 'formatted', statusm'old')

target - 'ingestsf.dfs'

call flleinl(fpath, target, nlen, olog, 41, prog) open (Ingx, file"fpath, access-'direct', recl-nlen, form 'formatted', status-'old')

target - 'Inhalesf.dfs'

call flleinlifpath, target, nlen, olog, 11, prog)

open(inhx, flle-fpath, access-'direct', recl-nlen, form-

'

target = 'submrsin.dfs

call fileini (fpath, target, nlen, olog, 11, prog)

open(131, Ille-fpath, access-'direct', recl-nlen, form'formatted', status='old'

cali filoins (f ath, targe

arget, nlen, olog, 11, progl

opentil, flle-fpath, access-'direct', recl-nlen, form-

'formatted', status='old' 
target-'grsurf00.dfs'

call fileini (fpath, target, nlen, olog, 11, prog) open (133, flle-fpath, accessm'direct', recl-nlen, form'formatted', statusw'old')

target - 'grvol 01.dfs'

call fileini (fpath, target, nlen, olog, 11, prog)

open (134, file-fpath, access='direct', recl-nlen, form: formatted', statusn'old's

target - 'grvol_05.dfs'

call fileini (fpath, target, nlen, olog, 11, prog)

open(135, file-fpath, access='direct', reci-nlen, form-

'cormatted, status='old's

target - 'grvol 15.dfs'

call flleini(fpath, target, nlen, olog, 41, prog)

open(136, file-fpath, access-'direct', recl-nlen, form'formatted', status='old')

target - 'grvolinf.dfs ?

call flleini(fpath, target, nlen, olog, 11, prog)

(acess-'direct', reci-nlen, form= : 'formatted', status='old's

return

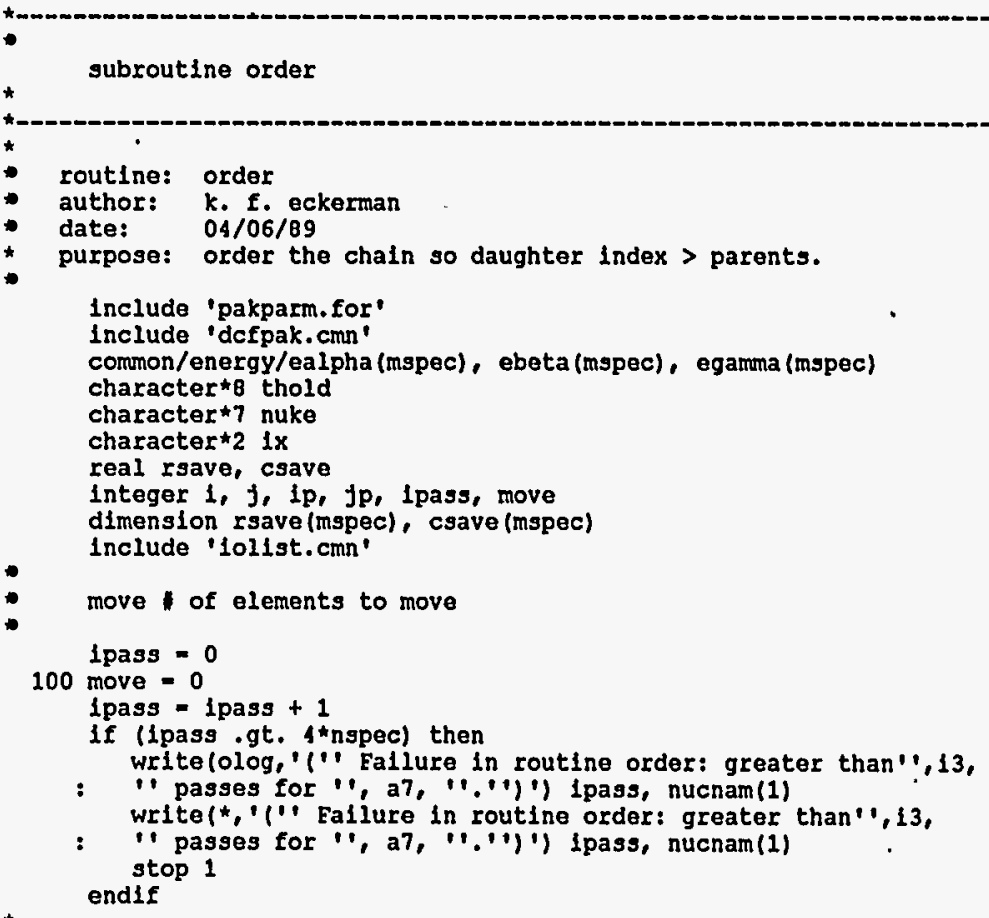

do $101=1$, nspec

do $10 \mathrm{~J}=1,1-1,1$, ne. 0.$)$ then

$$
\text { ip }=1
$$

Jp $=\mathrm{J}$

go to 15

endis

10 continue

if no elements to move then return

15 If (move .eq. 0) return

nuke - nucnam (1p)

thold - thalf (1p)

ea = ealpha (1p)

eg = egama (1p)

Ix $=1 \mathrm{u}(\mathrm{pp})$

do $20 \mathrm{f}-1$, nspec

20 conti 1

do $301-1$

do 301 - ip - 1, $f p_{0}-1$

nucnam $(1+1)-$ nucnam (1)

ealpha $(1+1)=$ ealpha(1)

ebeta $(1+1)$ - ebeta(1)

egamma $(1+1)$ - egamma(1)

1u $(1+1)=\operatorname{lu}(1)$

do $30 j-1$, nspec

30 continue

nucnam (Jp) = nuke

thalf (Jp) - thold

lu (jp) $=1 x$

ealpha (jp) = ea

ebeta (fp) $=-e b$

egamma (jp) - eg

branch $(j p, j)=$ rsave $(j)$

40 continue

do $501-1$, nspec

cgave $(1)$ - branch $(1,1 p)$

continue

do $60 f=1 p-1, j p,-1$

branch $(1, j+1)$ - branch $(1, j)$

60 continue

nspec

branch $(1, f p)$ - csave (1)
70 continue

Yes we do have backward polnt gotos In FORTRAN, sorry!

goto 100

end 
subroutine path

\section{author: K.E. Eckerman}

date: $04 / 06 / 09$

purpose: initialize

Adopted from A. Blrchall, Health Phys. 50, 3, 389-397, 1986.

Include 'pakparm. fox'

Include 'dcfpak.cmn'

Integer max, mpath

- comon/calcul/ max (mspec), mpath (mspec, mspec)

co 101 is napec

$\max (1)=0$
do $10 j-1$, ngpec
mpath $(1, j)=0$

10 continue

do $20 j=2$, nspec
do $201-1, j-1$

If (branch $(1, j)$, ne. 0.$)$ then

$$
\max (j)=\max (j)+1
$$

20 cond if

continue

retur
end.

\section{$\star$}

- subroutine pauseit

routine: pause

author: $K$.

date: $10 / 23 / 93$

purpose: pause w/o 11 ne feed

character $\star 1$ dumy

write $(*,(a))$, , HIt <Enter> to continue.'

read $(*, \cdot(b n, a 1)$ ') dumy

return subroutine printm

routine: printm

author: $k$. F. eckerman with modificatlons by f. c. ryman

date: $01 / 15 / 92$

purpose: print the decay chain

include 'pakparm.for'

Include 'dcfpak.cmn'

* local varlables.

character ${ }^{\star 7}$ nuke, rllst

(muke (mspec), rlist (mspec)

include 'lolist.cmn'

write(*, 8001)

Write(olog, 8001)

(nspec

write( $\$, 8002)$ nspec, nucnam(1), thalf(1), iu(1)

else

If (nucnam(1) $(: 2)$.eq. 'Sf') goto 20

nlist $=0$

do $10 \mathrm{f}-1$, nspec

If (1 . ne. $f$.and. branch $(1, j)$.ne. 0.0$)$ then

nlist - nlist +1

flist (n11st) $=1$

write (r11st (n11st), "(1pe7.1)") branch (1,j)

do $5 k=4,6$

rlist (nilst) $(k: k)$ - rlist (n11st) $(k+1: k+1)$ continue r11st (n11st) $(7: 7)=$.

write (*, 8002) 1, nucnam(1), thalf(1), iu(1),

(rilst(j), flist $(f)$, nuke(f), $f-1$, nilst) 2) 1, nuenam (1), thalf (1), iu(1),

continue

1f (nspec .gt. 5) pause
endif

- endil

clear screen and return

if (nspec .gt. 5) call cls

- formats

8001 format/' ', 3x, 'Nucllde Halfllfe f1',7x, 'Nucllde f2',7x, : 'Nuclide 3 $^{\prime}, 7 \mathrm{x}$, 'Nucllde'l 
8002 format (' ', 12,1x, a7, 1x, a8, a2, :, 3(1x,a6, '->',12,1x, a7))

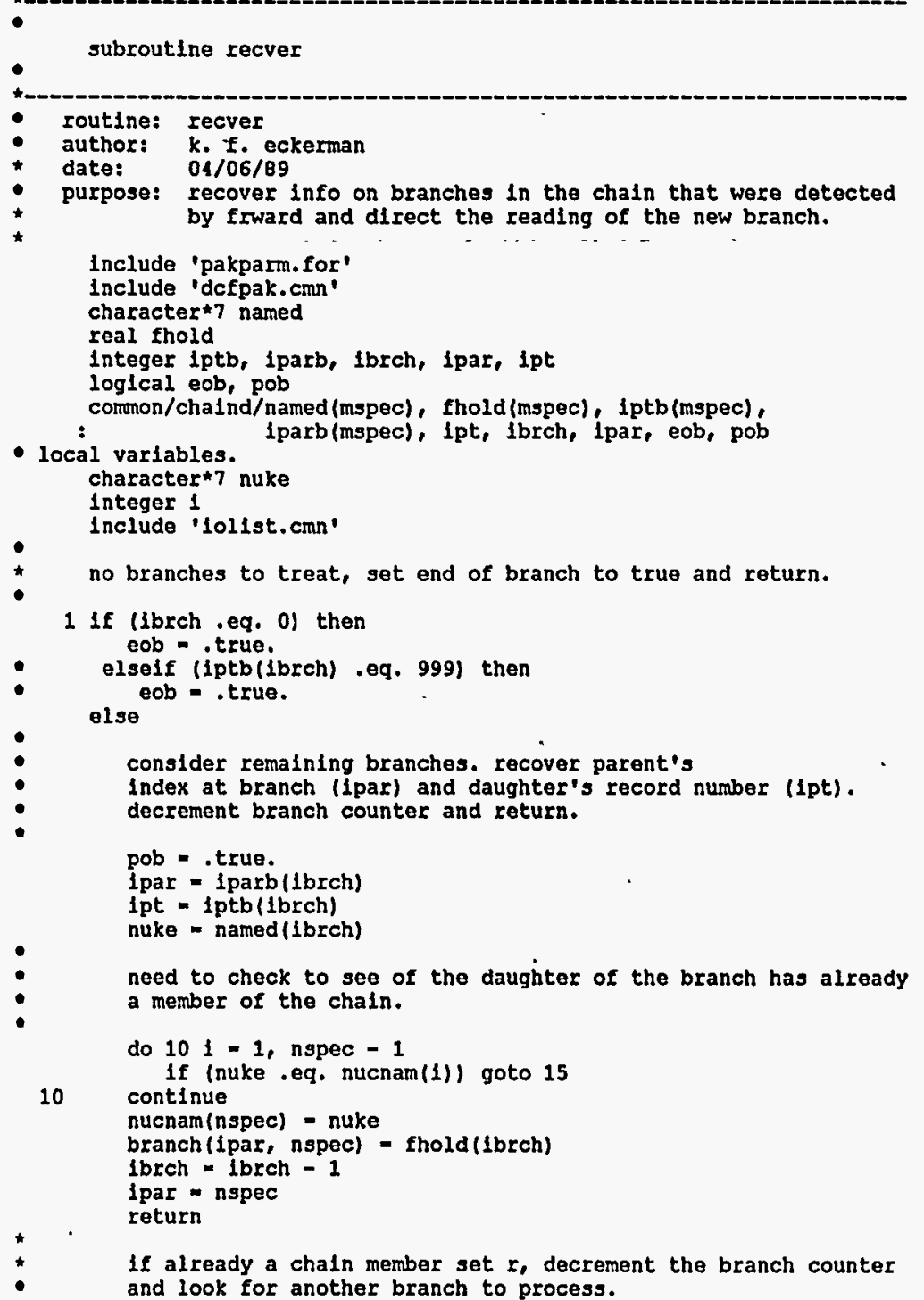

branch (1par,1) - fhold(1brch)

1brch - 1brch - 1

go to 1

- endis

- retur

end

subroutine sortem (ar, n)

routine: sortem

author: k.f. eckerman

(10)

a into descending numerical order

dimension ar( $(*)$

do $20 \mathrm{f}-2, \mathrm{n}$

do $11,1-1-1,1,-1$

If $(\operatorname{ar}(1)$. Ie. a) goto 12

$\operatorname{ar}(1+1)-\operatorname{ar}(1)$

contin

$12 \operatorname{ar}(1+1)=$

20 continue

return

end

subroutine nukeok (nuke, ok)

character $\$ 7$ nuke, check

character
logical ok

- function check ensure proper format of user input

nuke - check ( nuke )

call chekmn ( nuke)

* Eind the nuclide in the Index file

lpt - Iblnryl nuke)

If (ipt .eq. o) then

call chekab ( nuke )

end if

- If pointer 1pt is zero, the nuclide ls not ln the Index file

- else we have a valid nuclide to process

1f (lpt .eq. 0) then 


ok - . false.
else
ok - true.
end 1f
return
end

character $\star 2$ col

write (col, '(12.2) ") 1col

write (*, '(a))' ' '// char(27) // ' ['// col //'c'

retur

-

$\star$

subroutine curpos(1row, 1col)

author: k. f. eckerman

date: $12 / 08 / 93$

indicated row and column.

character*2 row, COl

write (row, $(12.2)$ ) lrow

writo(col, (12.2), 1001

else

col -101 '

end if

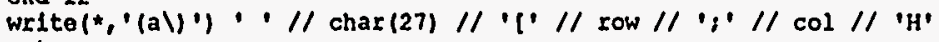
roturn end

3. Eunction Routines

character*(*) functlon check (nuke)

function: check

author: r.j. we

purpose: convert chemical symbol in nucllde name to proper

notation, $e, g, k r-85 m_{0}$ etc.

character*(*) nuke

- remove any leading blanks from nuke

nuke - 1 trim ( nuke)

- ensure first character is upper case.

if (nuke(:1) .ge, ' $a$ ' .and. nuke(:1) . le. ' 2 ')

: nuke = char(1char(nuke(:1)) - 32) // nuke(2:7)

- ensure second character, if present, 1s lower case.

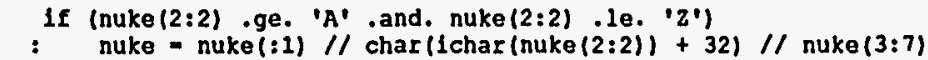


- ensure metastable notation, if present, is lower case.

do $30 \mathrm{~J}=4,7$

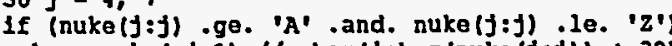

: nuke = nuke(:j-1) // char(1char(nuke(j:j)) + 32) // nuke(j+1:) continue

chec$$
\text { * }
$$

double precision function expf1 $(2 \mathrm{~m}, t)$

\section{$\begin{array}{ll}\text { author: } & \text { k. f. eckerman } \\ \text { date: } & 10 / 04 / 94\end{array}$}

purpose: routine to compute $[1.0-\exp (-1 \mathrm{~m} \cdot t)] / 1 \mathrm{~m}$.

double precision $1 m, t, 1 m t$, one, two, expfun, eps

logical first

parameter (one $-1.0 \mathrm{do}$, two $=2.0 \mathrm{~d} 0$ )

data flrst/ .true.

If (first) the

10 eps $=$ eps / two

if (eps + one .gt. one) goto 10

eps - dsqrt (eps)

end if $1 \mathrm{mt}=1 \mathrm{~m} \cdot \mathrm{t}$

If (1mt.1t. eps) then

expf1 $=t$ * (one $-1 m t /$ two)

else

expf1 = (one - expfun $(-1 m t)) / 1 m$

end if

return

$*$

double precision function expfun $(t)$

\section{author: $k$. f. eckerman}

date: $10 / 04 / 94$

purpose: routine to compute exp ( $t$ ).

double precision $t$, zero, upval

parameter (zero $=0.0 \mathrm{do}$, upval $-180 . \mathrm{d0}$ )

if (t. it. -upval) then

expfun - zero

else

expfun - dexp (t)

end if

retur

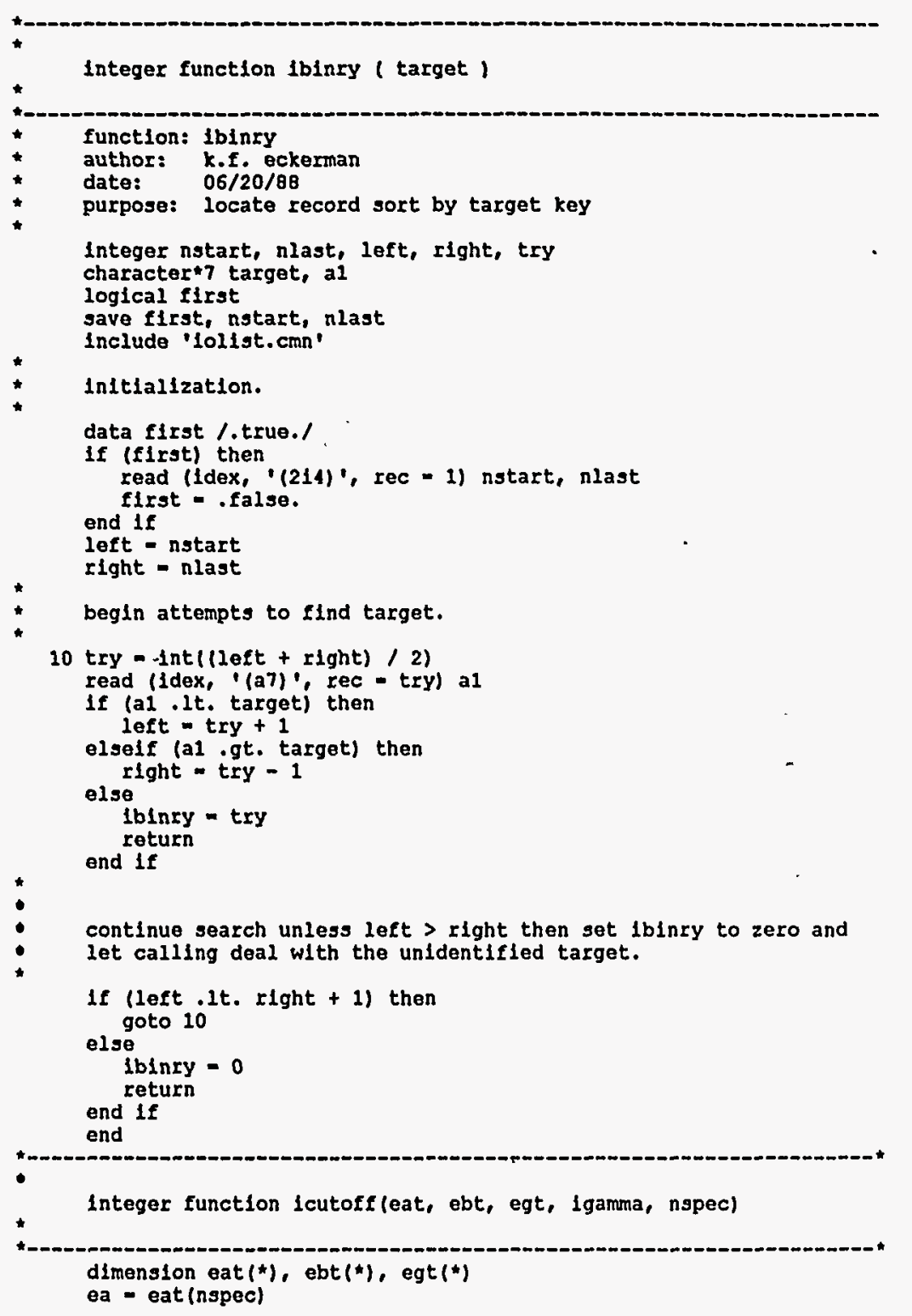


$e b-e b t$ (nspec)

If (ea (nspec) and, Igamma .eq. 0) then

do 101 - nspec- $1,1,-1$

If (eat (1) 1 1t. $0.99 * 0$ ) then

$$
\text { goto } 15
$$

10$$
\text { end if }
$$

continue

else

15 if (eb gt. 0.1 then

do 201 if (ebt (1).1t. $0.99^{-1}$ *b) then $1 \mathrm{~b}=1+$

$$
\text { goto } 25
$$

20$$
\text { continue }
$$

$b=1$

else

end if

25 if (eg.gt. 0.1 then
do 30 1 - nspec-1, $1,-1$.
If legt(i) .1t $1 g=1+$

30 end 11

continue

$1 \mathrm{ig}$

ig $=0$

35 1cutoff - maxo(19, maxo(1a, 1b)) return

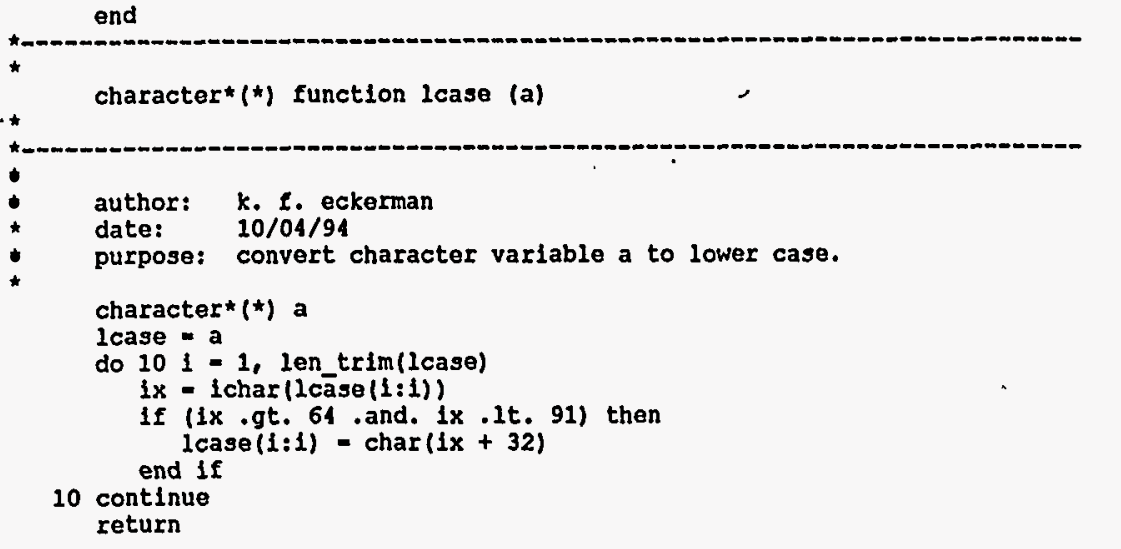

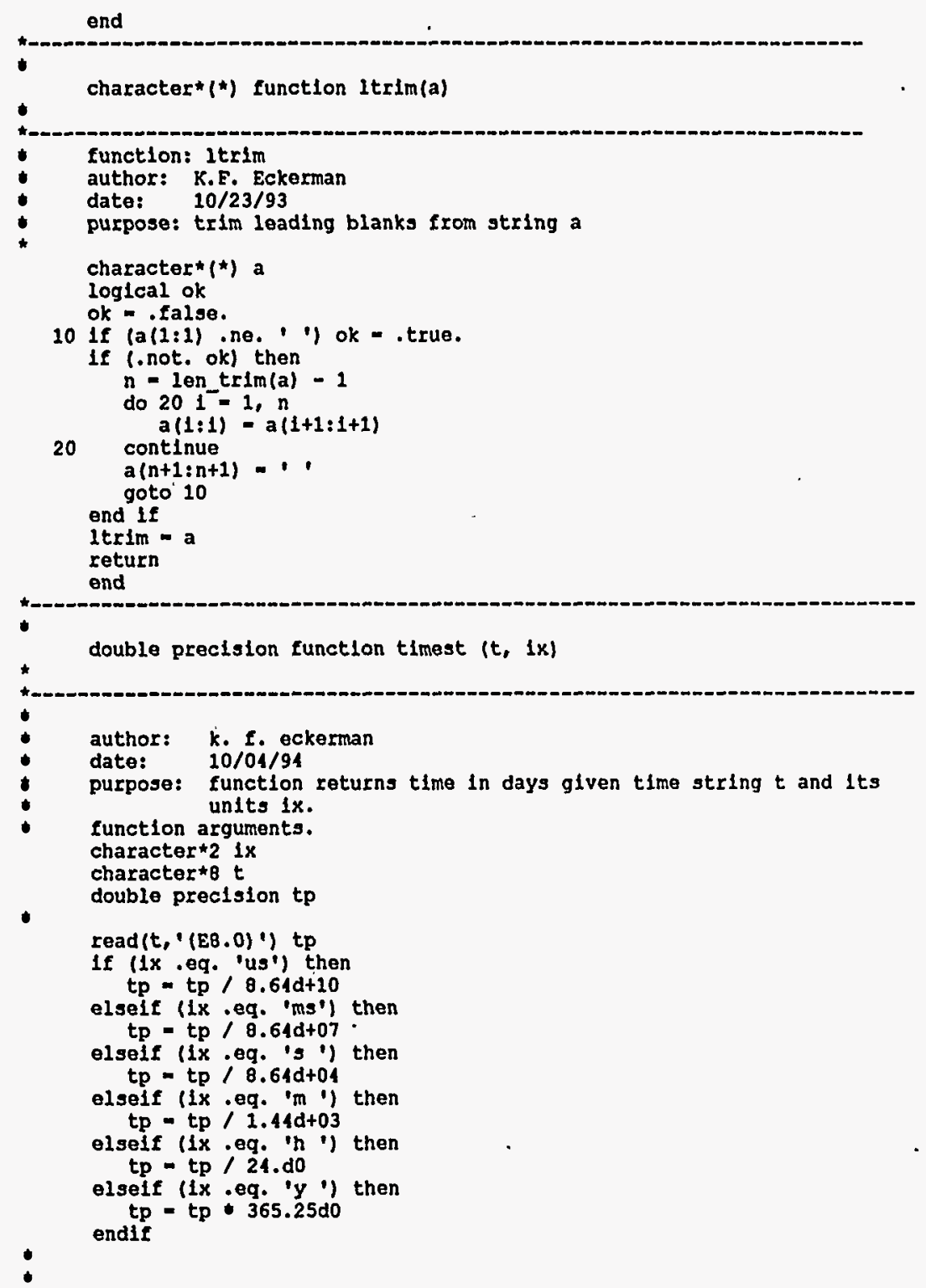


character*(*) function ucase (a)

\section{author: K. f. eckerman
date:}

purpose: convert character variable a to upper case.

character* $(*)$ a

ucase - a

do $101-1$, len_trim(ucase)

1x = 1char (ucase (1:1))

If (1x.gt. 96 .and. $1 x .1 \mathrm{t} .123)$ the

$$
\text { end if }
$$

10 continue

return

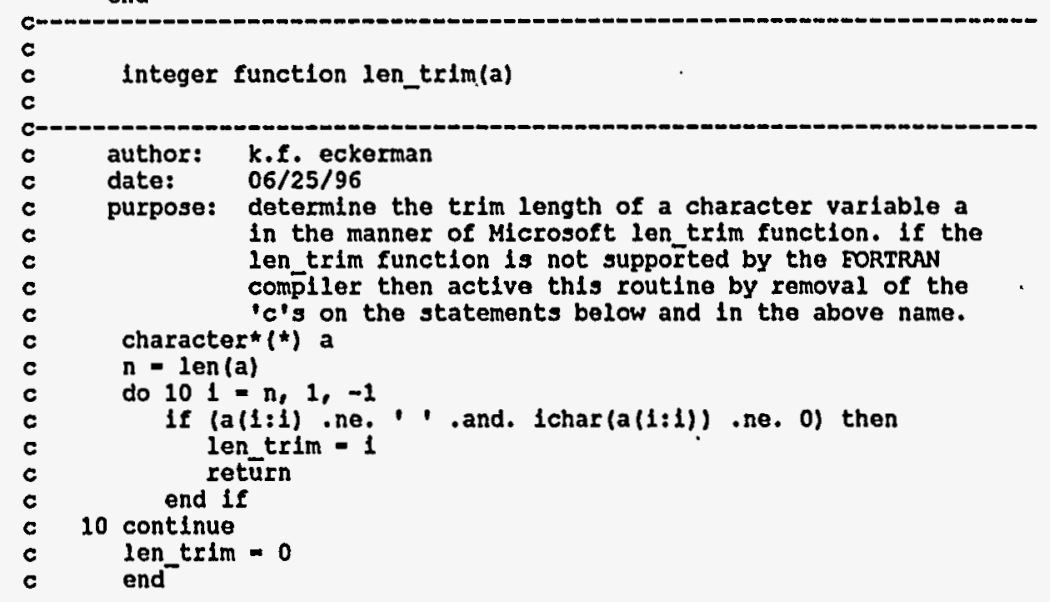

\section{program readem}

- READEM 11lustrates the manner-the electronic files of dose coefficients - for Sandia National Laboratory can be accessed in EORTRAN. The procedure uses the index file DEEXTINT. NDX to coordinate the reading of the coefficients from their respective flles.

The coefficient files are:

ICRPINGS.DAT - FGR 11/ICRP-30 ingestion intakes.

ICRPINS.DAT - EGR 11/CRP-30 Inhalation intakes.

FGR

EGR12E33.DAT - EGR 12 Table III.3 for ground surface contamination.

EGR12F34.DAT - EGR 12 Table III. 4 contaminated soll slab $1 \mathrm{~cm}$ thick.

FGR12F35.DAT - EGR 12 Table III.5 contaminated soll slab $5 \mathrm{~cm}$ thick.

FGR12E36.DAT - FGR 12 Table III.6 contaminated soll slab $15 \mathrm{~cm}$ thick.

EGR12E37.DAT - FGR 12 Table III. 7 contaminated soll infinite thlckness.

All flles are formatted direct access files. The RECL specification in - the FORTRAN open statement 1s read from the flle DCFPAK. INI. See that file * for further discussion regarding specifications for different compllers.

The flle FGR1112.IDX contalns the following information:

The flist record glves the record numbers of the flrst and last data

- record, format (214) which are 2 and 839 . The format of records 2 through - 839 is $(a 7, a 8, a 2, a 6,315,3(14,011.0), 3 f 7.0, e 11.0,1 x, a 9)$

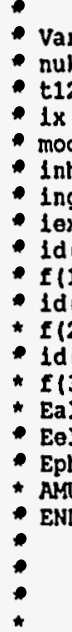

Variable Degcription

nuke

ix

- mode

inh

: lex

- id(1)

- Id(2)

* $E(2)$

- Id(3)

* E(3)

* Ealpha

- Eelectron

* AMU

ENDSE

Name of nuclide

Halflife

Halflife units

Record / for Inhalation

Record for ingestion

Record for external

Record of daughter

Branchling fraction

Record I of daughter

Branching fraction

Record of daughter

Branchlng fraction

Emitted alpha energy

Emitted electron energ

Emitted photon energy

Atomic mass

Eormat
$a 7$
$a 8$
$a 2$
$a 6$
$a 6$
15
15
15
14
e11
14
e11
14
$e 11$
57.0
67.0
17.0
e11
a9

author: k.f. eckerman

06/04/96:06/27/96

Include 'pakparm.for'

Include 'dcfpak.cmn'

Include 'batch.cmn'

character $\star 7$ nuke 
character*1 class, ucase

logical lpath (9), ok

dbatch - false.

numarg - nargs()

1f (numarg gt, 1) dbatch - .true.

this loop sets all pathway flags to trues $1 . e .$. we want dose cofficients for all pathways.

do $11=1$, 9

1 ipath(1) - .true.

call cls

write( $*, \cdot(1 x, "$ view dose coefficlents' ") ')

write $(*, \cdot(3 x, "$, K.F. Eckerman and R.w. Leggett '" $) ')$

write (*, , (3x, ", Oak RIdge National Laboratory', ')

* open direct access files and write text to screen

call openem

label 10 is the subject of a backward pointing goto

10 write(*, $\left.(a))^{\prime}\right)$ ' Input nucllde (e.g., Ba-137m or <Enter> to quit) $>1$ read $(*, \cdot(b n, a 7))^{\prime}$ nuke

If (len_trim(nuke) .ne. o) then

call nukeok to determine if nuke in data bases

call nukeok (nuke, ok)

15 (.not. ok) then

(.not. ok) then
write $\left(*, "\left(1 x_{1}(a), "\right.\right.$ is not in index (11el $\left.\left.\cdots\right) "\right)$ werte nukel:

write $(*, *)$

call pauselt

else

If no Inhalation class is Indicated then the class with the higest effective dose will be assumed.

class - '.

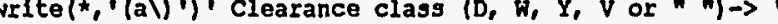
read $\left(*, '\left(b n_{,}\right.\right.$al) $)$class

class = ucase (class)

read in amad as a character and then do a internal read

write $(*, 1(a))$,$) , Input AMAD (default 1 \mu \mathrm{m}) \rightarrow$, read (*, ' (bn, a5)') amadx

else read(amadx, '(f5.0)') amad

end if

lets get some factors

flocal $=0.0$

call dosecof (nuke, floral, class, flinhl, amad, Ipath)

call tablem to print factors to screen

call tablem (amad)

endis

compute activity of chain members as at user specifled times call cls

- Activity and Integrated Activity of Chain Members write $(*, 1)$, $\left.(2))^{\prime}\right)$

: Input time of interest (d) : zero to quit $\rightarrow$,

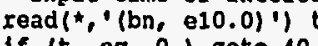

write $t_{1} \cdot 15 x_{0}+1$.

A(t)/AO IntA/AO(d) ' ')'

do over the maximum length of the retained chain members

do 30 1spec - $1, \max (n i n t$, next)

call birch (1spec, $t, a$, alnt)

act

30 continue thalf (1spec), 1u(1spec), a, alnt goto 20

* clear screen and goto 10 to get another nuclide.

call cls

- close all flles

call closem

end

subroutine tablem (amad)

routine: tablem

author: k.f. eckerman

purpose: write dose coefficients to screen.

include 'pakparm.for'

chclude defpak. can 
character 20 titles (mfact)

\section{character*18 units (mfact)}

character*8 $t$, ltrim, buffer

character $* 5$ buff$$
\text { Ground Surface }
$$

$\because$ 'Ingestion

$\because$ '1

is cal slab

soli 1

$\because$

'Infintte Thlck Soll '/

data units $/$ ' (Sv/Bg)

$\because$ ' (Sv/Bq)

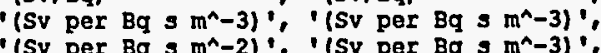

' (Sv per Bq $5 \mathrm{~m}^{n}-3$ ) $\therefore$, ' (Sv per Bq $=\mathrm{m}^{n}-3$ ) ?

do 100 lfact - 1 , mfact

If (1fact 10. 2) the

else$$
\text { nupper - next }
$$

end 1 do 50

ispec -1 , nupper

f (1flag (19pec, Ifact)) then

call cls

head - - Dose Coefflclents : // titles (Ifact) (:len_trim(

Ip - (80-1en trim(head) $) / 2$

call curpos $(\overline{2}, 1 p)$

write $(*, *)$ head(:Ien_trim(head))

write $(*, \bullet)$

$t$ - Itrim(Thalf(ispec))

call curright 6

(ifact eq. 1) then

write (buff5, ( $(15.2)$ ) ) amad
write $(*, *)$ nucnam(19pec) //

- T1/2 = // $t(:$ len_trim(t)) // . // iu(1spec) // ' Class: ' // classo (1spec)

11 I 1 -

elself (1fact .eq. 2) then

write(buffer, '(1pe8.1) 1) florl (19pec)

write $(*, *)$ nucnam (1spec)

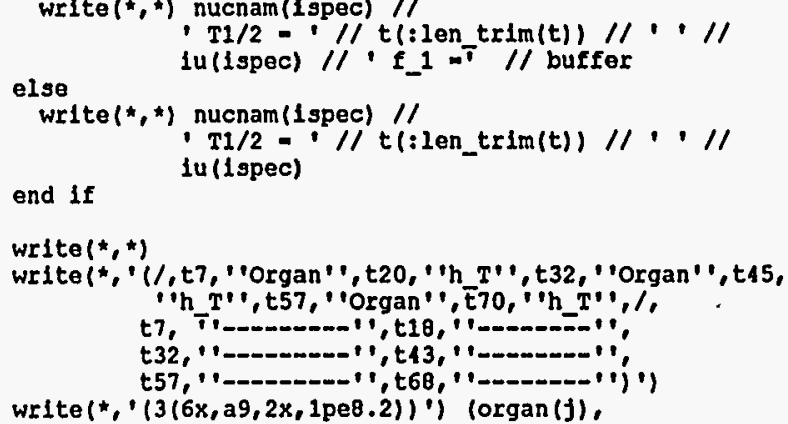

end if

write $(*, *)$

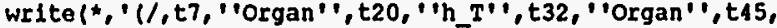

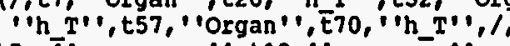

t7,

t32,

write (*, ' (3 (6x, 29, 2x, 1pe8.2)) if (organ(j).

: $\quad$ df(1spec, 1fact, $j), f-1,25)$

call curpos $(22,60)$

write (" ('Member: $" 1,12, "$ of $"(12) "$ ) 1spec, nupper end if

100 continue

return 


\section{INTERNAL DISTRIBUTION}

1. B. A. Berven

2. J. S. Bogard

3-7. K. F. Eckerman

8. C. E. Easterly

9. R. N. Hamm

10. G. D. Kerr

11. D. C. Kocher

12. R. W. Leggett

13. P. Y. Lu

14. C. A. Little

15. D. A. McLaughlin

16. J. C. Miller
17. S. T. Purucker

18. D. E. Reichle

19. P. S. Rohwer

20. J. C. Ryman

21. A. L. Sjoreen

22. R. C. Ward

23. Central Research Library

24. ORNL Y-12 Research Library

25. Laboratory Records Department

26. Laboratory Records Department (RC)

27. ORNL Patent Office

\section{EXTERNAL DISTRIBUTION}

28. Assistant Manager of Energy Research and Development, U.S. Department of Energy, Oak Ridge Operations Office, P.O. Box 2001, Oak Ridge, TN 37831-8600

29. B. B. Boecker, Inhalation Toxicology Research Institute, Lovelace Biomedical and Environmental Research Institute, P.O. Box 5890, Albuquerque, NM 87185

30. W. E. Bolch, 205 Nuclear Science Center, University of Florida, Gainesville, FL 32611

31. A. Bouville, National Cancer Institute, Radiation Effects Branch, Executive Plaza North, Suite 530, Bethesda, MD 20892

32-42. D. Clauss, Sandia National Laboratory, P.O. Box 5800, MS 0748, Albuquerque, NM 87185-0748

43. F. J. Congel, Incident Response Division, Office for Analysis and Evaluation of Operational Data, U.S. Nuclear Regulatory Commission, Washington, DC 20555

44. F. Harper, Sandia National Laboratory, P.O. Box 5800, MS-0748, Albuquerque, NM 87185-0748

45. C. B. Nelson, Office of Radiation and Indoor Air, U. S. Environmental Protection Agency, $401 \mathrm{M}$. Street, S. W., Mail Code 6602J, Washington, DC 20555

46. H. T. Peterson, Jr., Air, Water and Radiation Division, Office of Environmental Guidance, U. S. - Department of Energy, 1000 Independence Ave., SW, Washington, DC 20585

47. A. C. B. Richardson, Deputy Director for Federal Guidance Criteria and Standards Division, United States Environmental Protection Agency, Washington, DC 20460 
48. G. S. Roessler, Route 1, Box 139H, Elysian, MN 56028

49. . G. Runkle, U. S. Department of Energy, Albuquerque Operations, Environmental Safety and Health Division, Albuquerque, NM 87115

50. M. Smith, U.S. EPA/NAREL, 540 S. Morris Ave., Montgomery, AL 36115

51. A. Wallo, Air, Water and Radiation Division (EH-232), Office of Environmental Guidance, U. S. Department of Energy, 1000 Independence Ave., SW, Washington, DC 20585

52. W. Whicker, Professor, Department of Radiology and Health Sciences, Colorado State University, Fort Collins, CO 80523

53. S. S. Yaniv, Office of Nuclear Regulatory Research, U. S. Nuclear Regulatory Commission, Washington, DC 20555

54. M. Young, Sandia National Laboratory, P.O. Box 5800, MS-0748, Albuquerque, NM 87185-074ع

55-56. Office of Scientific and Technical Information, P.O. Box 62, Oak Ridge, TN 37831 